Article

\title{
Comparing World Economic and Net Energy Metrics, Part 3: Macroeconomic Historical and Future Perspectives
}

\author{
Carey W. King ${ }^{1,2}$ \\ Received: 3 March 2015 ; Accepted: 29 September 2015 ; Published: 17 November 2015 \\ Academic Editor: Robert Lundmark \\ ${ }^{1}$ Energy Institute, the University of Texas at Austin, 2304 Whitis Ave, C2400, Austin, TX 78712, USA; \\ careyking@mail.utexas.edu; Tel.: +1-512-471-5468 \\ 2 Jackson School of Geosciences, the University of Texas at Austin, 2275 Speedway, C9000, Austin, \\ TX 78712, USA
}

\begin{abstract}
I use energy cost share to characterize the role of energy in the economy. Specifically, I use an estimate of monetary expenditures for primary energy on an annualized basis for forty-four countries from 1978 to 2010 for natural gas, coal, petroleum, and electricity. I show that global energy cost share is significantly correlated to a one-year lag in the change in gross domestic product as well as measures of total factor productivity. Given the historical reduction in the relative cost of energy (including food and fodder for animate power) since the start of the Industrial Revolution, combined with a global energy cost share estimate, I conclude that the turn of the 21st Century represents the time period with the cheapest energy in the history of human civilization (to date). This potential historical nadir for energy expenditures around 2000 has important ramifications for strategies to solve future social, economic, and environmental problems such as reducing annual emissions of greenhouse gases (GHGs). Rapidly decreasing annual GHG emissions while internalizing their costs into the economy might feedback to increase energy expenditures to such a degree as to prevent economic growth during that transition.
\end{abstract}

Keywords: energy; net energy; economics; input-output; cost share; transition

\section{Introduction}

This manuscript is Part 3 of three papers comparing net energy and economic metrics. Each manuscript has similar background and motivation sections. Part 1 includes a fuller Background and Motivation before analyzing how net energy and power metrics translate to individual energy commodity (and technology) costs and prices, respectively [1]. Part 2 analyzes how net energy metrics translate to total expenditures on energy [2]. Part 3 places the calculations of expenditures on energy in historical, current, and future contexts.

\subsection{Background}

Considerable debate surrounds the role of energy in the economy and society, and much of the disagreement stems from different methodological approaches, models, paradigms, and time spans under consideration. Conclusions range from the possible unimportance of all resources [3], to energy price spikes being a chief determinant of recessions [4], to energy and prime-movers as being equally important to labor and capital in driving economic growth [5,6], to energy and prime-movers as the critical elements-more important than labor or capital—in driving economies during industrialization [7] and possibly over the long-term, [8-10]. Countries with high per capita 
gross domestic product (GDP) tend also to consume more primary energy per capita [11], even more so when considering energy embodied in imported products [12].

Access to a sufficient quantity of affordable energy and energy services is one of several important factors (also, for example, proper governance [13]) for modern living standards in an open industrial or post-industrial economy. To an economist, the prices and energy cost share are important metrics. To an ecologist and biophysical systems modeler, the energy return ratio (ERR) is an important metric for models and perspectives not purely based on monetary flows. ERRs are ratios of the energy delivered (or extracted) from an energy system divided by the energy invested to deliver that output.

While all models are wrong, some are useful. This statement is certainly poignant for energy, society, and the economy. Researchers consider the role of energy in society from multiple perspectives: anthropological [14-17], economic [4,7,8,10,18-21], ecological and biophysical $[9,11,22,23]$, and others. With these different perspectives come a multitude of quantitative and qualitative methods of analysis. These analyses try to answer questions related to how energy and natural resources influence and enable societal growth and structure.

While much research effort is spent on analyses to calculate ERRs of individual energy technologies and fuels for comparative analysis [24-33], there is a dearth of work linking individual ERRs to micro or macroeconomic metrics. Despite four decades since the development of the mathematical foundations in net energy analysis that relate economic accounts to country-level energy consumption [34,35], existing research has not effectively placed technology-specific net energy into the context of the broader macroeconomic modeling, economic projections, and energy-economic decision-making. This disconnect serves as motivation for this paper and other research of the corresponding author [24,36,37]. How do we relate economic and biophysical perspectives? How can we measure if affordability of energy were either acting as or indicating a constraint on economic production or growth? I contribute data and concepts to answer these two questions at the global scale and with a long-term perspective.

\subsection{Part 3 Content and Context}

The net energy and economy disconnect is particularly important in the context of recent discussions of a possible post-2008 "secular stagnation", reduction in the growth of various measures of innovation or productivity (e.g., total factor productivity). Summers [38] discusses how Japan's low-growth trajectory since 1990 is important to interpret for the US situation today. He also notes that losing $80 \%$ of electricity generation would collapse GDP, contrary to the common economic misperception that a small cost share for electricity (i.e., energy) means its cost cannot have but a small impact on GDP. Gordon [39] mentions six important "headwinds" for the United States economy (e.g., demographics, cost inflation for higher education, debt). The debt and population the world has accumulated did not occur overnight, just as the energy system did not develop overnight. In his US headwinds, Gordon includes "...environmental regulations and taxes ..." as a headwind, and thus it is important to understand the energy cost feedback associated with decarbonizing our energy system to mitigate climate change impacts. As described by systems approaches, these headwinds are expected to eventually occur at the global scale on a finite planet [40,41]. From the perspective of net energy and energy expenditures, how can we tell if they are a fundamental headwind? It is within the contexts of this paragraph in which I place the research of this manuscript.

This manuscript is organized as follows. Section 1.3 summarizes some of the relevant energy-related econometric literature. Then Section 2 describes the specific energy and economic calculations of this paper. Section 3.1 uses a simple correlation approach to suggest that world expenditures on energy are a relevant factor in describing economic growth. Section 4 provides context and interpretation of the long-term changes in net energy metrics (energy return ratios, power return ratios) and expenditures on energy, or energy cost share. Section 4.1 places the results into historical context of the Industrial Revolution and the transition to a fossil-fueled 
economy. The historical context shows that 10-15 years ago is likely the time of cheapest energy to date. Section 4.2 discusses the results in the context of internalizing $\mathrm{CO}_{2}$ emissions from fossil fuels implying that adherence to rapid emissions reductions targets might necessitate a quantity of energy-related expenditures historically associated with global recession. Finally, Section 5 concludes the paper.

\subsection{Summary of Econometric Perspectives}

Much of the research on energy-economic interactions is performed using econometric regression techniques. These techniques provide insight by relating historical energy costs to economic growth. For example, Hamilton indicates that short term increases in oil prices tend to precede US recessions [4,21]. Kilian stresses the importance of understanding oil prices in the context of broader macroeconomic fundamentals (e.g., aggregate demand for commodities due to global growth) in describing the global oil price rise from 2003 to 2008 primarily as a function of unexpected increasing demand (relative to supply) in emerging Asian economies (e.g., in China) [42]. Kopits [43] indicates there seems to be a carrying capacity, a threshold quantity of expenditures on oil relative to GDP, above which the US economy tends to be in recession. Bashmakov [44] explains that there is a critical threshold value (approximately $11 \%$ for OECD consumer expenditures on energy) that creates an increasingly negative impact on growth.

Kalimeris et al. [45] performed a meta-analysis of econometric studies that investigated the direction of causality between energy and GDP (e.g., does causality point from energy to GDP, GDP to energy, neither direction, or both directions). They conclude that their "... meta-analysis results (of 158 studies) neither support the existence of a fundamental 'macro' direction ..." nor the lack of causality between energy consumption and GDP. Much of this lack of unanimity can be attributed to the lack of any biophysical (e.g., laws of physics) framework. However, the subset of the Kalimeris et al. [45] studies that do support causality from energy to GDP account for energy quality in some way. These metrics of energy quality in one way or another account for "energy plus technology" rather than simply gross primary energy consumption. This quality factor is often one based upon relative prices of resources (e.g., the price per MJ is higher for oil than coal) [46], thermodynamic characterizations such as fuel exergy, technology conversion efficiency, and "useful work" delivered [5,6,10,18], or embodied energy concepts such as emergy [47,48]. The work of Ayres is particularly enlightening to show that energy, expressed as useful work (e.g., light, mechanical motion, heat, etc.), is indeed an important time-varying factor of production to include along with labor and capital. For example, Ayres and Voudouris [5] includes useful work to more accurately model the GDP of Japan, United States, and United Kingdom from 1900-2000. Some econometric studies investigating causal links of energy on GDP indicate that using longer time series and/or accounting for energy "quality" (either as an energy return ratio, energy cost share, or weighted by price) implies causality from energy to GDP [7,20,46,49-51], particularly during periods with increasing use of fossil fuels.

The econometric literature shows that it is valuable for econometric studies to have time series of prices and energy cost shares, or expenditures on energy as a fraction of income, GDP, or personal consumption expenditures $[4,7,19,20,46]$. This is because energy cost share serves to illuminate how low-cost energy relates, usually positively, to economic growth. As I discuss, energy cost shares for preindustrial economies were much higher than for industrial and post-industrial economies. Thus, the calculations of this paper have value in that they add new information on top of these energy-economic analyses by placing energy cost share of the contemporary economy in the context of a preindustrial economy and net energy of energy resources and technology. 


\section{Methods and Data}

\subsection{Correlation Analysis}

I conduct a very simple but insightful correlation of expenditures on energy as a fraction of GDP, $f_{e, \mathrm{GDP}}$, to the annual change in GDP and total factor productivity (TFP) by country and 44-country aggregate. Data for $f_{e, \mathrm{GDP}}$ come from Part 2 of this paper series [2]. For estimates of annual changes in TFP I use data from the Total Economy Database ${ }^{\mathrm{TM}}$ (TED) TFP for available countries from The Conference Board Total Economy Database ${ }^{\mathrm{TM}}$ [52], from 1990 to 2010 and OECD Multi-Factor Productivity (MFP) [53] from 1985 to 2010.

I recognize this is not a full econometric analysis, and that is left for future work.

\subsection{England and United Kingdom Data}

I use Roger Fouquet's collection of historical data on England and United Kingdom energy prices and primary energy supply to estimate expenditures on primary energy [54-57]. The GDP data come from [58] for 1300-1700, and for post-1700 data are from Mitchell [59] and the UK Office of National Statistics. These data, provided via Fouquet, enabled calculation of the historical energy cost share data presented in Section 3.2.

\subsection{United States Data}

I use data from Table 2.3.5 of the United States Bureau of Economic Analysis (BEA) to show an estimate of personal food and energy expenditures as a fraction of US GDP [60]. The BEA describes the nominal personal consumption expenditures for "energy goods and services" as those that "Consists of gasoline and other energy goods and of electricity and gas." For food and food services expenditures I add the two BEA line items of "Food and beverages purchased for off-premises consumption" and "Food services and accommodations". Nominal GDP data are from the St. Louis Federal Reserve data code (time series) A191RC1.

I also use data from the BEA summary input-output tables to estimate intermediate purchases by food and natural resource extraction sectors (e.g., not personal consumption as a component of GDP). This calculation provides a more consistent comparison to the calculation of country and world $f_{e, \text { GDP }}$ with both [2] and the data from Fouquet for England and the UK I define food and energy sectors as the following. Standard Industrial Classification summary sectors = Iron and ferroalloy ores mining; nonferrous metal ores mining; coal mining; crude petroleum and natural gas; stone and clay mining and quarrying; chemical and fertilizer mineral mining; petroleum refining and related industries; private electric, gas, water, and sanitation services; livestock and livestock products; other agricultural products; forestry and fishery products; agricultural, forestry, and fishery services; and food and kindred products; tobacco manufacturers. North American Industry Classification System summary sector codes = oil and gas extraction; mining, except oil and gas; support activities for mining; utilities; petroleum and coal products; farms; forestry, fishing, and related activities, food and beverage and tobacco products; and food and beverage stores; food services and drinking places.

\section{4. $\mathrm{CO}_{2}$ Emissions from Energy Consumption}

I estimate a monetary penalty for carbon dioxide, $\mathrm{CO}_{2}$, emissions due to fossil fuel combustion. The emissions data for each of the 44 countries analyzed in this manuscript come from the IEA website [62]. This provides a consistent set of input data since the calculation of expenditures on energy, $f_{e, \mathrm{GDP}}$, for these 44 countries originates from the IEA data set [2].

\section{Results}

The major results are as follows, with more detail in the rest of this section: 
- Global expenditures on energy expressed as a fraction of GDP is significantly correlated with the one-year lag of the annual changes in both GDP and total factor productivity, but not with zero-year lag.

- The one-year lag correlation is statistically significant only when including expenditures for oil and when considering oil expenditures only.

\subsection{Correlation of $f_{e, G D P}$ to GDP and Total Factor Productivity}

Here I present the results for the simple but insightful correlation of $f_{e, G D P}$ to annual change in both GDP $(\triangle \mathrm{GDP})$ and TFP $(\triangle \mathrm{TFP})$ by country and 44 country aggregate. I do this for two calculations of expenditures on energy. The "Actual Data" calculation is based upon using data in the IEA data without replacing missing energy price data, and the "Estimated" calculation is when substituting non-zero values for missing price data (see King et al. [2]).

There are no significant correlations of Estimated $f_{e, \mathrm{GDP}}$ to annual change in either $\Delta \mathrm{GDP}$ or $\Delta \mathrm{TFP}$ with a 0 -year lag. Applying a 1-year lag, however, to $\Delta \mathrm{GDP}$ (Table 1) and $\Delta \mathrm{TFP}$ (Table 2) does produce interesting correlations. The correlation is weaker for lags greater than one year, and I do not show these results. The countries with statistically significant $(p$-value $<0.05)$ negative linear Pearson correlations between 1-year lagged annual change in either GDP or TFP with Estimated $f_{e, G D P}$ are:

- for 1-year lagged $\triangle$ GDP: United Kingdom, Spain, The Netherlands, Sweden, Greece, Nigeria, and Portugal (see Table 1).

- for 1-year lagged $\triangle$ TFP from TED: United States, Russia, Canada, France, Sweden, and Greece (see Table 2).

- for 1-year lagged $\triangle$ MFP from OECD: The Netherlands, Canada, France, Sweden, and South Korea (see Table 2).

Aucott and Hall [61] performed a similar calculation as in Table 1 for the US from 1950 to 2013. They found a significant negative correlation between $f_{e, \text { GDP }}$ and both $\Delta$ GDP and 1-year lagged $\triangle$ GDP (but slightly higher for a 0-year lag), whereas my US negative correlation is not statistically significant at 0-year lag. Three of the four listed PIIGS countries (Portugal, Greece, and Spain) have significant negative correlation of $f_{e, \mathrm{GDP}}$ to lagged $\Delta \mathrm{GDP}$, and those three countries have had substantial economic difficulty since the start of the Great Recession in late 2007.

One outlier from the correlations is Qatar. Qatar, a major natural gas exporter, has a statistically significant positive correlation $(r=0.36)$ of $f_{e, G D P}$ with 1-year lagged GDP growth. A more thorough analysis is needed to determine the causation of the Qatari correlation, but one hypothesis is that recessions related to high oil expenditures translate to higher natural gas consumption and /or natural gas prices linked to rising oil prices that inherently drive up Qatari GDP.

I also examined correlations of $f_{e, G D P}$ to $\triangle \mathrm{GDP}$ and $\triangle \mathrm{TFP}$ for the 44 country "worldwide" aggregate defined by only those countries for which GDP and TFP data exist. The "worldwide" correlation of Estimated $f_{e, \mathrm{GDP}}$ with 1-year lagged $\triangle \mathrm{GDP}$ has a correlation coefficient of approximately $r=-0.45$ and $p$-value $=0.01$ (see Table 1) and for no lag, $r=-0.26$ and $p$-value $=0.14$ (not significant). For the same 1-year lagged correlation to $\triangle \mathrm{TFP}$ (TED) and $\triangle \mathrm{MFP}$ (OECD), the correlation coefficients are $r=-0.49$ and $r=-0.51$ with $p$-values 0.029 and 0.009 , respectively (Table 2). Thus, the correlation of world $f_{e, \text { GDP }}$ to 1-year lag of annual changes in both GDP and TFP change is statistically significant but not to annual change of GDP or TFP in the same year.

I also investigated correlating oil expenditures only. The Estimated only oil $f_{e, \mathrm{GDP}}$ (including net imports of refined oil) is significantly negatively correlated with 1-year lagged $\Delta$ GDP change for eight countries as well as for the world aggregate (see Table 3). The correlation of 1-year lagged $\Delta T F P$ to only oil $f_{e, \mathrm{GDP}}$ is statistically significant for 12 countries (either TED or OECD estimates) as opposed to eight when correlating to all energy (Table 4). The negative correlation of "worldwide" 1 -year lagged $\triangle \mathrm{TFP}$ to oil only $f_{e, \mathrm{GDP}}$ is also statistically significant with approximately the same correlation coefficient $(r=-0.52 \mathrm{OECD} ; r=-0.48 \mathrm{TED})$ as for Estimated total world expenditures on energy. 
The correlations of 1-year lagged $\Delta \mathrm{TFP}$ with Estimated $f_{e, \mathrm{GDP}}$ considering all expenditures on energy except for oil reveal only five countries with statistically significant negative correlations (USA, Greece, Nigeria, and Poland using the TED TFP as well as South Korea using the OECD MFP) (see Table 5). The "worldwide" $\Delta$ TFP negative correlations with $f_{e, \mathrm{GDP}}$ for non-oil expenditures are not statistically significant when considering either OECD MFP data or TED TFP data. The higher correlation of $f_{e, \mathrm{GDP}}$ to economic growth factors when including oil agrees with the widely-held finding that oil is the most economically important energy commodity (see Figure 3 and [2], indicating that oil expenditures are dominant).

Table 1. The negative correlation of the ratio of GDP for all energy expenditures to the 1 year lag in annual change in GDP is statistically significant for several countries with the most severe economic stress, and statistically significant for the world overall. ${ }^{*}=p$ value $<0.05 ;{ }^{* *}=p$ value $<0.01$. The countries in this table account for approximately $93 \%-95 \%$ of world GDP.

\begin{tabular}{|c|c|c|c|c|}
\hline \multirow{2}{*}{ Country } & \multicolumn{2}{|c|}{$\%$ of GDP on Energy (Actual Data) } & \multicolumn{2}{|c|}{$\%$ of GDP on Energy (Estimated) } \\
\hline & $\begin{array}{l}\text { Correlation } \\
\text { Coefficient }\end{array}$ & $p$ Value & $\begin{array}{l}\text { Correlation } \\
\text { Coefficient }\end{array}$ & $p$ Value \\
\hline USA & -0.263 & 0.145 & -0.284 & 0.115 \\
\hline UK & -0.289 & 0.109 & -0.451 & $0.010^{* *}$ \\
\hline SPAIN & -0.463 & $0.008^{* *}$ & -0.623 & $0.0001^{* *}$ \\
\hline RUSSIA & 0.121 & 0.508 & -0.0397 & 0.829 \\
\hline NETHERLANDS & -0.494 & $0.004^{* *}$ & -0.452 & $0.010 * *$ \\
\hline JAPAN & 0.073 & 0.69 & 0.14 & 0.443 \\
\hline ITALY & -0.472 & $0.006^{*}$ & -0.327 & 0.068 \\
\hline GERMANY & 0.12 & 0.513 & 0.102 & 0.578 \\
\hline CANADA & -0.359 & $0.044^{*}$ & -0.319 & 0.075 \\
\hline FRANCE & -0.514 & $0.003^{* *}$ & -0.323 & 0.071 \\
\hline AUSTRIA & -0.35 & $0.049^{*}$ & -0.337 & 0.060 \\
\hline DENMARK & -0.273 & 0.13 & -0.303 & 0.092 \\
\hline FINLAND & -0.129 & 0.481 & -0.0398 & 0.829 \\
\hline NORWAY & -0.382 & $0.031 *$ & 0.127 & 0.487 \\
\hline SWEDEN & -0.469 & $0.007 * *$ & -0.362 & $0.042 *$ \\
\hline ARGENTINA & 0.411 & $0.019^{*}$ & 0.09 & 0.624 \\
\hline AUSTRALIA & -0.185 & 0.311 & -0.237 & 0.192 \\
\hline BELGIUM & -0.587 & $0.0004^{* *}$ & -0.328 & 0.066 \\
\hline BRAZIL & -0.133 & 0.468 & -0.038 & 0.838 \\
\hline CHINA & 0.359 & $0.044 *$ & -0.129 & 0.481 \\
\hline TAIPEI & -0.515 & 0.003 ** & 0.104 & 0.57 \\
\hline COLOMBIA & 0.063 & 0.732 & -0.019 & 0.918 \\
\hline $\mathrm{CZECH}$ & 0.167 & 0.361 & 0.058 & 0.751 \\
\hline GREECE & -0.439 & 0.012 * & -0.452 & $0.010^{* *}$ \\
\hline HUNGARY & -0.389 & $0.028 *$ & -0.194 & 0.287 \\
\hline INDIA & 0.46 & $0.008^{* *}$ & 0.138 & 0.452 \\
\hline INDONESIA & -0.089 & 0.628 & 0.097 & 0.598 \\
\hline IRAN & 0.246 & 0.175 & -0.010 & 0.956 \\
\hline IRAQ & 0.347 & 0.052 & 0.265 & 0.142 \\
\hline S KOREA & -0.434 & 0.013 * & -0.291 & 0.107 \\
\hline KUWAIT & -0.197 & 0.279 & -0.293 & 0.104 \\
\hline LIBYA & 0.112 & 0.541 & 0.179 & 0.328 \\
\hline MALAYSIA & -0.24 & 0.186 & -0.215 & 0.238 \\
\hline MEXICO & -0.184 & 0.314 & -0.195 & 0.285 \\
\hline NEW ZEALAND & -0.082 & 0.655 & -0.206 & 0.258 \\
\hline NIGERIA & 0.143 & 0.436 & -0.377 & $0.033 *$ \\
\hline
\end{tabular}


Table 1. Cont.

\begin{tabular}{lcccc}
\hline \multirow{2}{*}{ Country } & \multicolumn{2}{c}{$\%$ of GDP on Energy (Actual Data) } & \multicolumn{2}{c}{ \% of GDP on Energy (Estimated) } \\
\cline { 2 - 5 } & $\begin{array}{l}\text { Correlation } \\
\text { Coefficient }\end{array}$ & $p$ Value & $\begin{array}{c}\text { Correlation } \\
\text { Coefficient }\end{array}$ & $p$ Value \\
\hline POLAND & 0.085 & 0.645 & -0.302 & 0.093 \\
PORTUGAL & -0.497 & $0.004^{* *}$ & -0.394 & $0.026^{*}$ \\
QATAR & 0.478 & $0.006^{* *}$ & 0.373 & $0.036^{*}$ \\
SAUDI ARABIA & -0.101 & 0.581 & -0.133 & 0.467 \\
S AFRICA & 0.087 & 0.635 & -0.017 & 0.926 \\
SWITZERLAND & -0.090 & 0.625 & -0.032 & 0.864 \\
TURKEY & -0.035 & 0.85 & -0.215 & 0.237 \\
VENEZUELA & 0.252 & 0.164 & -0.083 & 0.65 \\
\hline WORLD & -0.369 & $0.038^{*}$ & -0.447 & $0.010^{*}$ \\
\hline
\end{tabular}

Table 2. The correlation between 1-year lagged change in total factor productivity (measured as logarithmic differences) and $f_{e, \mathrm{GDP}}$ for all energy for both the TED TFP and OECD MFP is statistically significant for several countries and the world overall. Results using Estimated $f_{e, \mathrm{GDP}}$ should be viewed as more representative because some Actual data $f_{e, \mathrm{GDP}}$ have missing data. ${ }^{*}=p$ value $<0.05$; ** $=p$ value $<0.01$. The countries with TFP in the Total Economy Database ${ }^{\mathrm{TM}}$ account for $89-92 \%$ of the world GDP from 1990-2010. The countries with MFP in the OECD account for $61 \%-75 \%$ of the world GDP from 1985-2010 (66\% in 1985 rising to $75 \%$ in 1995 then declining to $66 \%$ in 2009 and $61 \%$ in 2010).

\begin{tabular}{|c|c|c|c|c|c|c|c|c|}
\hline \multirow[b]{3}{*}{ Country } & \multicolumn{4}{|c|}{ TED TFP } & \multicolumn{4}{|c|}{ OECD MFP } \\
\hline & \multicolumn{2}{|c|}{ Actual } & \multicolumn{2}{|c|}{ Estimated } & \multicolumn{2}{|c|}{ Actual } & \multicolumn{2}{|c|}{ Estimated } \\
\hline & $\begin{array}{l}\text { Correlation } \\
\text { Coefficient }\end{array}$ & $p$ Value & $\begin{array}{l}\text { Correlation } \\
\text { Coefficient }\end{array}$ & $p$ Value & $\begin{array}{l}\text { Correlation } \\
\text { Coefficient }\end{array}$ & $p$ Value & $\begin{array}{l}\text { Correlation } \\
\text { Coefficient }\end{array}$ & $p$ Value \\
\hline USA & -0.565 & $0.009 * *$ & -0.545 & $0.011 *$ & -0.306 & 0.137 & -0.306 & 0.137 \\
\hline UK & -0.456 & $0.043 *$ & -0.416 & 0.068 & -0.432 & 0.035 * & -0.389 & 0.060 \\
\hline SPAIN & -0.315 & 0.176 & -0.317 & 0.173 & -0.047 & 0.825 & -0.065 & 0.757 \\
\hline JAPAN & -0.317 & 0.173 & -0.104 & 0.663 & -0.215 & 0.301 & -0.207 & 0.321 \\
\hline ITALY & -0.216 & 0.361 & -0.314 & 0.178 & -0.249 & 0.230 & -0.360 & 0.077 \\
\hline GERMANY & -0.075 & 0.753 & -0.107 & 0.654 & -0.245 & 0.327 & -0.273 & 0.272 \\
\hline CANADA & -0.650 & $0.002 * *$ & -0.642 & $0.002 * *$ & -0.464 & $0.019 *$ & -0.491 & $0.013^{*}$ \\
\hline FRANCE & -0.616 & $0.004^{* *}$ & -0.635 & $0.003^{* *}$ & -0.577 & $0.003^{* *}$ & -0.461 & 0.020 * \\
\hline AUSTRIA & -0.155 & 0.514 & -0.159 & 0.504 & -0.191 & 0.512 & -0.237 & 0.415 \\
\hline SWEDEN & -0.456 & $0.043 *$ & -0.490 & $0.028 *$ & -0.491 & 0.013 * & -0.418 & $0.037^{*}$ \\
\hline ARGENTINA & 0.049 & 0.837 & 0.008 & 0.974 & - & - & - & - \\
\hline AUSTRALIA & 0.083 & 0.727 & -0.324 & 0.163 & -0.059 & 0.790 & -0.287 & 0.184 \\
\hline BELGIUM & -0.411 & 0.072 & -0.352 & 0.128 & -0.423 & 0.035 * & -0.330 & 0.107 \\
\hline BRAZIL & 0.068 & 0.775 & -0.239 & 0.311 & - & - & - & - \\
\hline CHINA & -0.063 & 0.793 & 0.048 & 0.842 & - & - & - & - \\
\hline TAIPEI & - & - & - & - & - & - & - & - \\
\hline COLOMBIA & 0.015 & 0.952 & -0.039 & 0.872 & - & - & - & - \\
\hline CZECH & -0.078 & 0.766 & 0.002 & 0.995 & - & - & - & - \\
\hline GREECE & -0.431 & 0.058 & -0.451 & $0.046^{*}$ & - & - & - & - \\
\hline HUNGARY & -0.404 & 0.077 & -0.416 & 0.068 & - & - & - & - \\
\hline INDIA & 0.115 & 0.630 & 0.002 & 0.994 & - & - & - & - \\
\hline
\end{tabular}


Table 2. Cont.

\begin{tabular}{|c|c|c|c|c|c|c|c|c|}
\hline \multirow[b]{3}{*}{ Country } & \multicolumn{4}{|c|}{ TED TFP } & \multicolumn{4}{|c|}{ OECD MFP } \\
\hline & \multicolumn{2}{|c|}{ Actual } & \multicolumn{2}{|c|}{ Estimated } & \multicolumn{2}{|c|}{ Actual } & \multicolumn{2}{|c|}{ Estimated } \\
\hline & $\begin{array}{l}\text { Correlation } \\
\text { Coefficient }\end{array}$ & $p$ Value & $\begin{array}{l}\text { Correlation } \\
\text { Coefficient }\end{array}$ & $p$ Value & $\begin{array}{l}\text { Correlation } \\
\text { Coefficient }\end{array}$ & $p$ Value & $\begin{array}{l}\text { Correlation } \\
\text { Coefficient }\end{array}$ & $p$ Value \\
\hline LIBYA & - & - & - & - & - & - & - & - \\
\hline MALAYSIA & -0.105 & 0.658 & -0.072 & 0.762 & - & - & - & - \\
\hline MEXICO & -0.348 & 0.133 & -0.349 & 0.132 & - & - & - & - \\
\hline NEW ZEALAND & -0.431 & 0.058 & -0.417 & 0.067 & -0.169 & 0.420 & -0.144 & 0.493 \\
\hline NIGERIA & -0.159 & 0.504 & -0.024 & 0.920 & - & - & - & - \\
\hline POLAND & -0.277 & 0.237 & 0.245 & 0.297 & - & - & - & - \\
\hline PORTUGAL & -0.024 & 0.920 & -0.046 & 0.846 & -0.236 & 0.397 & -0.262 & 0.345 \\
\hline QATAR & -0.039 & 0.869 & 0.030 & 0.901 & - & - & - & - \\
\hline SAUDI ARABIA & -0.290 & 0.215 & -0.268 & 0.254 & - & - & - & - \\
\hline S AFRICA & -0.145 & 0.542 & -0.297 & 0.204 & - & - & - & - \\
\hline SWITZERLAND & -0.162 & 0.496 & -0.147 & 0.536 & -0.076 & 0.757 & -0.056 & 0.819 \\
\hline TURKEY & -0.192 & 0.417 & -0.214 & 0.365 & - & - & - & - \\
\hline VENEZUELA & 0.051 & 0.831 & -0.017 & 0.942 & - & - & - & - \\
\hline WORLD & -0.531 & $0.016^{*}$ & -0.490 & $0.028 *$ & -0.520 & $0.008^{* *}$ & -0.509 & $0.009 * *$ \\
\hline
\end{tabular}

Table 3. The correlation of the ratio of GDP for oil only energy expenditures, both Actual Data expenditures using available data and Estimated expenditures when substituting missing price data, to the 1-year lagged annual change in GDP shows less negative correlation to individual countries and the world overall than to the ratio of all energy expenditures to GDP (see Table 1). ${ }^{*}=p$ value $<0.05 ;{ }^{* *}=p$ value $<0.01$. The countries in this table account for approximately $93 \%-95 \%$ of world GDP.

\begin{tabular}{|c|c|c|c|c|}
\hline \multirow{2}{*}{ Country } & \multicolumn{2}{|c|}{$\%$ of GDP on Oil (Actual Data, 1-Year Lag) } & \multicolumn{2}{|c|}{$\%$ of GDP on Oil (Estimated, 1-Year Lag) } \\
\hline & $\begin{array}{l}\text { Correlation } \\
\text { Coefficient }\end{array}$ & $p$ Value & $\begin{array}{l}\text { Correlation } \\
\text { Coefficient }\end{array}$ & $p$ Value \\
\hline USA & -0.296 & 0.112 & -0.364 & $0.041 *$ \\
\hline UK & -0.314 & 0.0905 & -0.451 & $0.010^{* *}$ \\
\hline SPAIN & -0.572 & $0.001^{* *}$ & -0.524 & $0.002 * *$ \\
\hline RUSSIA & -0.050 & 0.794 & 0.055 & 0.766 \\
\hline NETHERLANDS & -0.533 & $0.002^{* *}$ & -0.39 & 0.027 * \\
\hline JAPAN & 0.121 & 0.523 & 0.145 & 0.427 \\
\hline ITALY & -0.189 & 0.316 & -0.045 & 0.806 \\
\hline GERMANY & -0.196 & 0.3 & -0.169 & 0.354 \\
\hline CANADA & -0.322 & 0.0829 & -0.272 & 0.132 \\
\hline FRANCE & -0.476 & $0.008^{* *}$ & -0.234 & 0.198 \\
\hline AUSTRIA & -0.461 & $0.010 *$ & -0.376 & 0.034 * \\
\hline DENMARK & -0.106 & 0.577 & -0.112 & 0.543 \\
\hline FINLAND & -0.141 & 0.459 & 0.007 & 0.971 \\
\hline NORWAY & -0.196 & 0.3 & -0.035 & 0.849 \\
\hline SWEDEN & -0.228 & 0.225 & -0.189 & 0.301 \\
\hline ARGENTINA & 0.445 & $0.014 *$ & -0.092 & 0.616 \\
\hline AUSTRALIA & -0.263 & 0.161 & -0.224 & 0.218 \\
\hline BELGIUM & -0.565 & $0.001 * *$ & -0.311 & 0.084 \\
\hline BRAZIL & 0.174 & 0.358 & 0.024 & 0.898 \\
\hline CHINA & 0.282 & 0.132 & -0.163 & 0.371 \\
\hline TAIPEI & -0.495 & $0.005^{* *}$ & 0.15 & 0.412 \\
\hline COLOMBIA & -0.003 & 0.987 & -0.104 & 0.57 \\
\hline $\mathrm{CZECH}$ & 0.238 & 0.206 & -0.119 & 0.517 \\
\hline GREECE & -0.434 & 0.017 * & -0.432 & 0.014 * \\
\hline HUNGARY & -0.298 & 0.109 & -0.105 & 0.566 \\
\hline INDIA & 0.383 & 0.037 * & 0.145 & 0.43 \\
\hline INDONESIA & -0.052 & 0.787 & 0.113 & 0.539 \\
\hline IRAN & 0.118 & 0.534 & -0.083 & 0.652 \\
\hline IRAQ & 0.398 & 0.029 * & 0.268 & 0.138 \\
\hline S KOREA & -0.66 & $7.0 \times 10^{-05 * *}$ & -0.183 & 0.317 \\
\hline
\end{tabular}


Table 3. Cont.

\begin{tabular}{lcccc}
\hline \multirow{2}{*}{ Country } & \multicolumn{2}{c}{ \% of GDP on Oil (Actual Data, 1-Year Lag) } & \% of GDP on Oil (Estimated, 1-Year Lag) \\
\cline { 2 - 5 } & $\begin{array}{l}\text { Correlation } \\
\text { Coefficient }\end{array}$ & $\boldsymbol{p}$ Value & $\begin{array}{c}\text { Correlation } \\
\text { Coefficient }\end{array}$ & $p$ Value \\
\hline KUWAIT & -0.178 & 0.347 & -0.29 & 0.107 \\
LIBYA & 0.056 & 0.769 & 0.163 & 0.371 \\
MALAYSIA & -0.255 & 0.173 & -0.223 & 0.22 \\
MEXICO & -0.065 & 0.734 & -0.145 & 0.427 \\
NEW ZEALAND & -0.090 & 0.637 & -0.133 & 0.468 \\
NIGERIA & 0.219 & 0.246 & -0.413 & $0.019^{*}$ \\
POLAND & -0.034 & 0.86 & -0.257 & 0.156 \\
PORTUGAL & -0.456 & $0.011^{*}$ & -0.362 & $0.042^{*}$ \\
QATAR & 0.36 & 0.051 & 0.412 & $0.019^{*}$ \\
SAUDI ARABIA & -0.235 & 0.21 & -0.158 & 0.388 \\
S AFRICA & 0.106 & 0.576 & 0.031 & 0.865 \\
SWITZERLAND & -0.118 & 0.533 & -0.161 & 0.961 \\
TURKEY & -0.103 & 0.589 & -0.163 & 0.378 \\
VENEZUELA & 0.172 & 0.363 & -0.364 & 0.373 \\
\hline WORLD & -0.469 & $0.009 * *$ & $0.040^{*}$ \\
\hline
\end{tabular}

Table 4. The correlation between 1-year lagged change in total factor productivity (measured as logarithmic differences) and $f_{e, \text { GDP }}$ on oil only for both the TED TFP and OECD MFP calculations. The negative correlation is statistically significant for several countries and the world overall. Results using Estimated $f_{e, \mathrm{GDP}}$ should be viewed as more representative because some Actual data $f_{e, \mathrm{GDP}}$ have missing data. ${ }^{*}=p$ value $<0.05 ;{ }^{* *}=p$ value $<0.01$. The countries with TFP in the Total Economy Database $\mathrm{T}^{\mathrm{TM}}$ account for $89 \%-92 \%$ of the world GDP from 1990 to 2010 . The countries with MFP in the OECD account for $61 \%-75 \%$ of the world GDP from 1985 to 2010 (66\% in 1985 rising to $75 \%$ in 1995 then declining to $66 \%$ in 2009 and $61 \%$ in 2010).

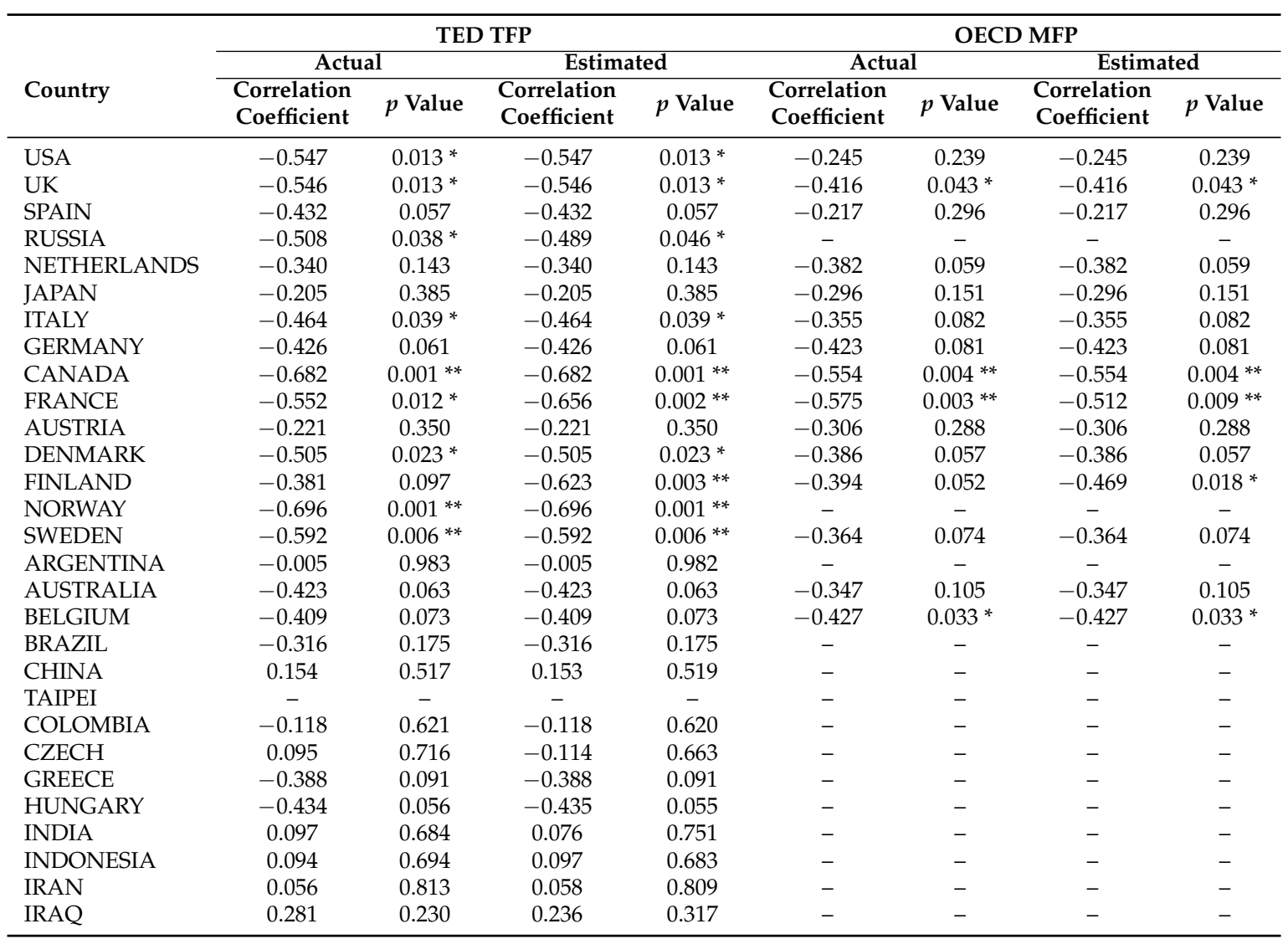


Table 4. Cont.

\begin{tabular}{|c|c|c|c|c|c|c|c|c|}
\hline \multirow[b]{3}{*}{ Country } & \multicolumn{4}{|c|}{ TED TFP } & \multicolumn{4}{|c|}{ OECD MFP } \\
\hline & \multicolumn{2}{|c|}{ Actual } & \multicolumn{2}{|c|}{ Estimated } & \multicolumn{2}{|c|}{ Actual } & \multicolumn{2}{|c|}{ Estimated } \\
\hline & $\begin{array}{l}\text { Correlation } \\
\text { Coefficient }\end{array}$ & $p$ Value & $\begin{array}{l}\text { Correlation } \\
\text { Coefficient }\end{array}$ & $p$ Value & $\begin{array}{l}\text { Correlation } \\
\text { Coefficient }\end{array}$ & $p$ Value & $\begin{array}{l}\text { Correlation } \\
\text { Coefficient }\end{array}$ & $p$ Value \\
\hline S KOREA & -0.147 & 0.537 & -0.275 & 0.241 & -0.513 & $0.009 * *$ & -0.402 & $0.046^{*}$ \\
\hline KUWAIT & -0.185 & 0.436 & -0.104 & 0.662 & - & - & - & - \\
\hline LIBYA & - & - & - & - & - & - & - & - \\
\hline MALAYSIA & -0.115 & 0.628 & -0.101 & 0.671 & - & _- & - & - \\
\hline MEXICO & -0.340 & 0.142 & -0.340 & 0.142 & - & - & - & - \\
\hline NEW ZEALAND & -0.331 & 0.154 & -0.331 & 0.154 & -0.120 & 0.566 & -0.120 & 0.566 \\
\hline NIGERIA & 0.071 & 0.768 & 0.072 & 0.764 & - & - & - & - \\
\hline POLAND & -0.280 & 0.233 & -0.429 & 0.059 & - & - & - & - \\
\hline PORTUGAL & -0.204 & 0.388 & -0.204 & 0.388 & -0.364 & 0.182 & -0.364 & 0.182 \\
\hline QATAR & -0.037 & 0.879 & -0.144 & 0.546 & - & - & - & - \\
\hline SAUDI ARABIA & -0.352 & 0.128 & -0.288 & 0.219 & - & - & - & - \\
\hline S AFRICA & -0.229 & 0.331 & -0.229 & 0.331 & - & - & - & - \\
\hline SWITZERLAND & -0.164 & 0.488 & -0.164 & 0.488 & -0.132 & 0.591 & -0.132 & 0.591 \\
\hline TURKEY & -0.290 & 0.215 & -0.290 & 0.215 & - & - & - & - \\
\hline VENEZUELA & -0.067 & 0.780 & -0.067 & 0.779 & - & - & - & - \\
\hline WORLD & -0.479 & $0.033 *$ & -0.483 & $0.031 *$ & -0.542 & $0.005^{* *}$ & -0.524 & 0.007 ** \\
\hline
\end{tabular}

Table 5. The correlation between 1-year lagged change in total factor productivity (measured as logarithmic differences) and $f_{e, G D P}$ on all energy except oil for both the TED TFP and OECD MFP calculations. The negative correlation is statistically signficant for a few countries but not the world overall. Results using Estimated $f_{e, \mathrm{GDP}}$ should be viewed as more representative because some Actual data $f_{e, \mathrm{GDP}}$ have missing data. ${ }^{*}=p$ value $<0.05$; ${ }^{* *}=p$ value $<0.01$. The countries with TFP in the Total Economy Database ${ }^{\mathrm{TM}}$ account for $89 \%-92 \%$ of the world GDP from 1990 to 2010 . The countries with MFP in the OECD account for 61\%-75\% of the world GDP from 1985 to 2010 (66\% in 1985 rising to $75 \%$ in 1995 then declining to $66 \%$ in 2009 and $61 \%$ in 2010 ).

\begin{tabular}{|c|c|c|c|c|c|c|c|c|}
\hline \multirow[b]{3}{*}{ Country } & \multicolumn{4}{|c|}{ TED TFP } & \multicolumn{4}{|c|}{ OECD MFP } \\
\hline & \multicolumn{2}{|c|}{ Actual } & \multicolumn{2}{|c|}{ Estimated } & \multicolumn{2}{|c|}{ Actual } & \multicolumn{2}{|c|}{ Estimated } \\
\hline & $\begin{array}{l}\text { Correlation } \\
\text { Coefficient }\end{array}$ & $p$ Value & $\begin{array}{l}\text { Correlation } \\
\text { Coefficient }\end{array}$ & $p$ Value & $\begin{array}{l}\text { Correlation } \\
\text { Coefficient }\end{array}$ & $p$ Value & $\begin{array}{l}\text { Correlation } \\
\text { Coefficient }\end{array}$ & $p$ Value \\
\hline USA & -0.481 & $0.032 *$ & -0.478 & $0.033 *$ & -0.366 & 0.072 & -0.366 & 0.072 \\
\hline UK & -0.295 & 0.206 & -0.205 & 0.385 & -0.337 & 0.107 & -0.258 & 0.223 \\
\hline SPAIN & 0.035 & 0.884 & -0.037 & 0.877 & 0.334 & 0.103 & 0.231 & 0.267 \\
\hline JAPAN & -0.091 & 0.704 & 0.183 & 0.440 & 0.126 & 0.550 & 0.186 & 0.373 \\
\hline ITALY & -0.076 & 0.749 & -0.204 & 0.389 & -0.157 & 0.455 & -0.310 & 0.131 \\
\hline GERMANY & 0.053 & 0.826 & 0.017 & 0.943 & -0.114 & 0.653 & -0.157 & 0.533 \\
\hline CANADA & -0.498 & 0.025 * & -0.403 & 0.078 & -0.241 & 0.247 & -0.243 & 0.242 \\
\hline FRANCE & -0.456 & 0.043 * & -0.397 & 0.083 & -0.298 & 0.148 & -0.221 & 0.289 \\
\hline SWEDEN & -0.379 & 0.100 & -0.322 & 0.166 & -0.451 & 0.024 * & -0.286 & 0.166 \\
\hline ARGENTINA & 0.283 & 0.227 & 0.023 & 0.925 & - & - & - & - \\
\hline AUSTRALIA & 0.597 & $0.005^{* *}$ & -0.043 & 0.859 & 0.250 & 0.250 & -0.039 & 0.860 \\
\hline BELGIUM & -0.292 & 0.211 & -0.149 & 0.532 & -0.179 & 0.391 & -0.020 & 0.923 \\
\hline BRAZIL & 0.251 & 0.286 & 0.017 & 0.942 & - & - & - & - \\
\hline CHINA & -0.143 & 0.548 & -0.010 & 0.967 & - & - & - & - \\
\hline TAIPEI & - & - & - & - & - & - & - & - \\
\hline COLOMBIA & 0.337 & 0.146 & 0.142 & 0.551 & - & - & - & - \\
\hline $\mathrm{CZECH}$ & -0.330 & 0.196 & 0.055 & 0.835 & - & - & - & - \\
\hline GREECE & -0.606 & $0.005^{* *}$ & -0.595 & $0.006^{* *}$ & - & - & - & - \\
\hline HUNGARY & -0.159 & 0.504 & -0.381 & 0.097 & - & - & - & - \\
\hline INDIA & 0.132 & 0.580 & -0.165 & 0.487 & - & - & - & - \\
\hline
\end{tabular}


Table 5. Cont.

\begin{tabular}{lcccccccc}
\hline & \multicolumn{4}{c}{ TED TFP } & \multicolumn{4}{c}{ OECD MFP } \\
\cline { 2 - 9 } Country & \multicolumn{2}{c}{ Actual } & \multicolumn{3}{c}{ Estimated } & \multicolumn{2}{c}{ Actual } & \multicolumn{2}{c}{ Estimated } \\
\cline { 2 - 9 } & $\begin{array}{c}\text { Correlation } \\
\text { Coefficient }\end{array}$ & $p$ Value & $\begin{array}{c}\text { Correlation } \\
\text { Coefficient }\end{array}$ & $p$ Value & $\begin{array}{c}\text { Correlation } \\
\text { Coefficient }\end{array}$ & $p$ Value & $\begin{array}{c}\text { Correlation } \\
\text { Coefficient }\end{array}$ & $p$ Value \\
\hline S KOREA & 0.046 & 0.846 & -0.254 & 0.279 & 0.115 & 0.583 & -0.441 & $0.028^{*}$ \\
KUWAIT & 0.052 & 0.829 & -0.338 & 0.145 & - & - & - & - \\
LIBYA & - & - & - & - & - & - & - & - \\
MALAYSIA & 0.116 & 0.627 & 0.051 & 0.832 & - & - & - & - \\
MEXICO & -0.335 & 0.149 & -0.350 & 0.130 & - & - & - & - \\
NEW ZEALAND & -0.424 & 0.063 & -0.381 & 0.097 & -0.158 & 0.451 & -0.109 & 0.604 \\
NIGERIA & -0.177 & 0.455 & -0.569 & $0.009 * *$ & - & - & - & - \\
POLAND & -0.021 & 0.930 & 0.476 & $0.034 *$ & - & - & - & - \\
PORTUGAL & 0.196 & 0.408 & 0.212 & 0.370 & -0.044 & 0.878 & -0.064 & 0.821 \\
QATAR & 0.000 & 0.999 & 0.271 & 0.248 & - & - & - & - \\
SAUDI ARABIA & 0.319 & 0.170 & -0.037 & 0.876 & - & - & - & - \\
S AFRICA & 0.177 & 0.455 & -0.383 & 0.096 & - & - & - & - \\
SWITZERLAND & -0.013 & 0.956 & 0.004 & 0.987 & 0.044 & 0.859 & 0.075 & 0.760 \\
TURKEY & -0.125 & 0.598 & -0.138 & 0.561 & - & - & - & - \\
VENEZUELA & 0.307 & 0.188 & 0.245 & 0.297 & - & - & - & - \\
\hline WORLD & -0.372 & 0.106 & -0.412 & 0.071 & -0.261 & 0.207 & -0.310 & 0.132 \\
\hline
\end{tabular}

\subsection{England and the United Kingdom}

From 1300 to 1800 the pre-industrial English economy typically spent between $30 \%$ and $40 \%$ equivalent of its GDP for energy, $f_{e, \mathrm{GDP}}$, as input for providing energy services (see Figure 1) [56,57]. The two time periods (early 1300s and early 1600s) with $f_{e, \mathrm{GDP}}>40 \%$ correspond to times of high population pressure relative to native food supply [17]. From 1300 to 1800, the economy grew at a slow rate of $<1 \% /$ year for both real GDP/year and real GDP/person/yr $[39,54,57]$. England (and the UK) $f_{e, \text { GDP }}$ did not fall below $25 \%$ until the 1830s. The percentage of the England/UK energy supply derived from biomass energy (e.g., food for labor, fodder for animals, and wood) was $>70 \%$ before 1600 and dropped steadily to $13 \%$ by 1830 [55]. During this time the absolute energy consumed via biomass increased, but coal consumption increased at a much more rapid rate, thus taking over the majority of the primary energy mix. After the 1830s $f_{e, \mathrm{GDP}}$ dropped quickly for 90 years through World War I, below $10 \%$, as the benefits accumulated from investments associated with the Industrial Revolution and fossil fuel consumption.

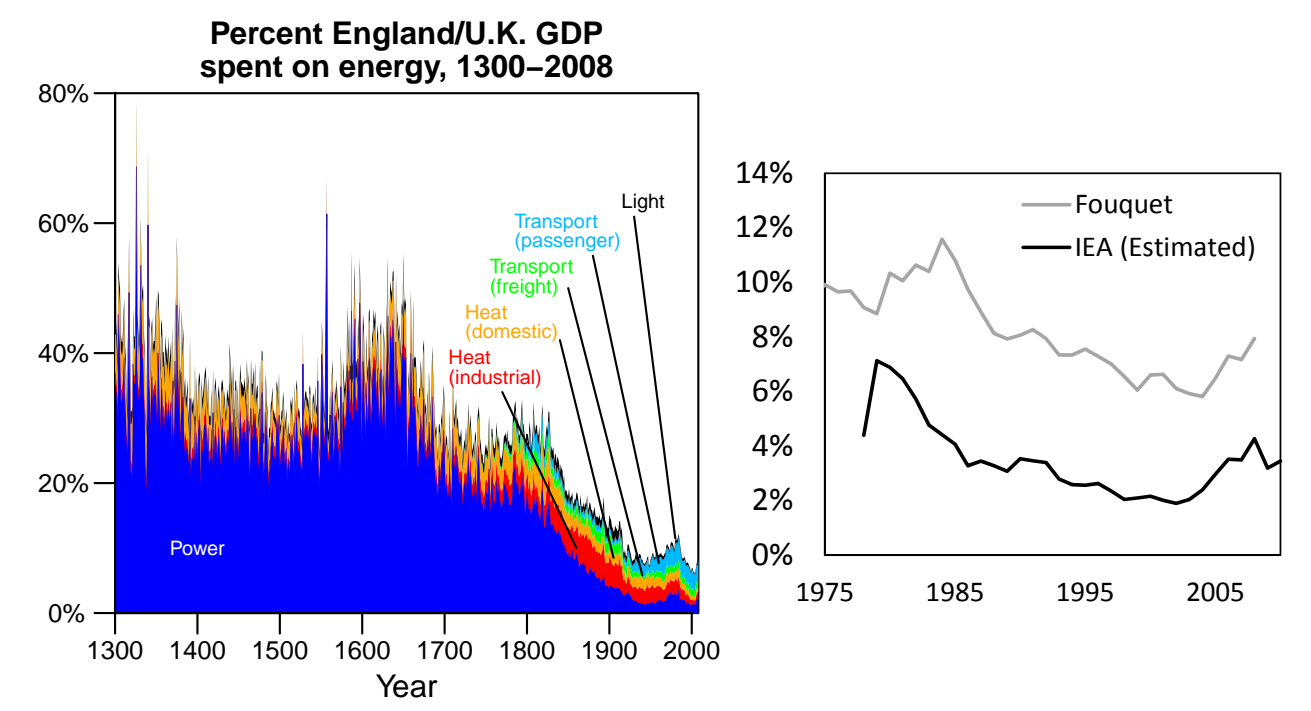

Figure 1. The plot represents $f_{e, \mathrm{GDP}}$ for purchasing energy for services of industrial power, industrial heating, domestic heating, freight transport, passenger transport, and lighting. England and UK data come from Roger Fouquet [57]. IEA estimated $f_{e, \mathrm{GDP}}$ come from King et al. [2]. 
Preindustrial England was severely power-limited because of reliance on biomass-fueled animate power to work the land in producing solar-powered stocks of food and fodder (see [55] for historical England and UK energy mix data). The percent of England/UK GDP spent on energy, $f_{e, \mathrm{GDP}}$, shows that the industrial (fossil fuel) era is distinct in having less than $15 \%$ of GDP allocated to purchase energy. In Figure 1 the lowest $f_{e, \mathrm{GDP}}$ is $6.2 \%$ in 2004 rising to $8.6 \%$ in 2008 (last data in 2008). The calculations of this paper using IEA data are significantly lower than Fouquet's calculations. For example, I estimate the UK's lowest $f_{e, \mathrm{GDP}}$ for primary energy at $1.9 \%$ in 2002 . The differences are largely due to different boundary conditions, in that I attempt to characterize primary energy whereas Fouquet primarily calculates energy spending at the consumer and domestic level. Nonetheless, the trends of my and Fouquet's calculations are very similar from 1978 to 2010.

\subsection{The United States}

In the United States, the post World War II era is characterized by a continuous decline in relative food costs until 2006, and a decline in "food + energy" costs from 1945 until 2002. Relative to GDP, US food and energy costs combined were never cheaper than 2002. This trend holds whether considering consumer expenditures on food and energy relative to GDP (lines in Figure 2) or intermediate spending by the food, energy, and resource sectors (circles in Figure 2), both of which were near $13 \%$ in 2012.

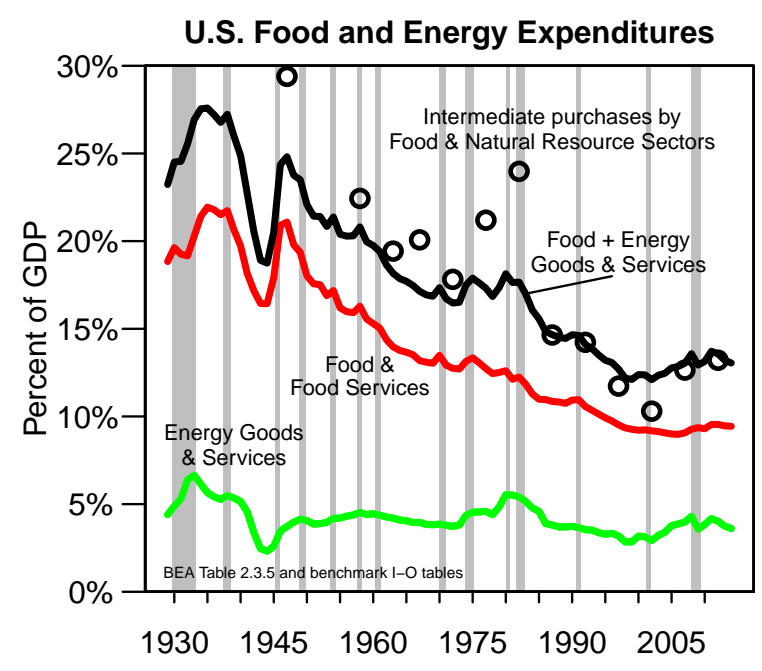

Figure 2. The quantity of personal consumption expenditures (PCE) for food and energy services in the United States as a fraction of US gross domestic product from 1929 to 2014. The gray bars represent time periods of US recession. The data plotted as lines are personal consumption expenditures (components of GDP) from Table 2.3.5 of the Bureau of Economic Analysis [60]. The data as circles represent intermediate purchases (not components of part of GDP) by food and energy sectors from harmonized BEA input-output tables.

\section{Discussion}

My relatively simple correlation analysis of the aggregated world expenditures on energy confirms that the relative cost of energy is an important factor in explaining TFP, and thus GDP growth. Further, these results suggest, but do not prove, a causal effect of monetary energy expenditures on economic growth and TFP (see Tables 1,2), but that conclusion needs further investigation via a full econometric analysis and other methods. The country-specific significant correlations of $f_{e, \mathrm{GDP}}$ to 1-year lagged $\triangle \mathrm{GDP}$ and $\triangle \mathrm{TFP}$ are limited to seven and eight countries, respectively, that are primarily OECD countries. Further, because the 44-country aggregate $f_{e, \mathrm{GDP}}$, $\triangle \mathrm{GDP}$, and $\triangle \mathrm{TFP}$ are weighted by GDP, countries such as the United States affect it more than countries with smaller GDP. Interestingly, despite this weighting, the United States, with the 
largest GDP, did not have a significant negative correlation of $f_{e, \mathrm{GDP}}$ to 1-year lagged $\Delta \mathrm{GDP}$, while the 44-country aggregate did have a significant correlation. Thus, in order to have a significant energy-economic relationship at the global scale it is not necessary to have significant relationships within a majority of the countries.

The IEA data are more complete for OECD countries, and thus, for these reasons mentioned in the previous paragraph, the results in this paper are largely reflective of developed countries. Future work can make increased use of the data sets and incorporate more variables to relate $f_{e, G D P}$ to other factors (e.g., labor, capital) considered to model GDP and energy relationships in previous studies, particularly those of those of Ayres, Voudouris, Kümmel, and Stern [5,6,8,10,18,49,51]. For example, Ayres and Voudouris [5] show that for the US, the UK, and Japan, production functions including capital, labor, and "useful energy" are more accurate than those assuming only inputs of capital and labor. Voudouris et al. [6] analyze $15 \mathrm{EU}$ countries (13 of which are in my data IEA data set) using the method of Ayres and Voudouris [5]. Interestingly, the average marginal product (e.g., contribution to economic growth) of "useful energy" is near zero for Ireland, the Netherlands, Portugal, and Spain; and the latter three (Ireland is not in my data set) have significant correlations of $f_{e, \mathrm{GDP}}$ to 1-year lagged $\Delta \mathrm{GDP}$. While I analyze more countries as well as a world aggregate, albeit in a more limited manner, I come to the same basic conclusion as the studies referenced in this paragraph: energy metrics and concepts describe a significant portion of macroeconomic growth and total factor productivity. Thus, it is crucial to include energy-related metrics to properly define what we mean when using the word technology to describe economic growth (e.g., technology for converting fuels into mechanical power and useful work versus technology for other purposes such as information).

\subsection{Historical Context of Expenditures on Energy}

The time series of the United Kingdom, Sweden, and the United States help to place the calculation of the more recent 44 -country aggregate $f_{e, \mathrm{GDP}}$ in long-term historical context. The United Kingdom (UK) was the first industrialized nation to make considerable use of fossil fuels (coal) to power industrial machinery (see Figure 1) and substitute for animate power. It is reasonable to assume that in the 1800s no country spent less on energy, relative to GDP. Thus, data on England and the UK provide insight into how expenditures on energy correlate with the transition to fossil fuels with high ERRs. For comparison, Kander also shows that Sweden's energy cost share of the economy (including food and fodder for animate power) consistently declined from $90 \%$ in the early 1800 s to less than 20\% after 1925 as Sweden shifted from biomass to coal $[7,20]$. Data from the US Bureau of Economic Analysis also show that US "food + energy" expenditures as a fraction of GDP declined from the 1930s until 2002, primarily driven by technology that enabled cheaper food (see Figure 2).

Considering the calculated $f_{e, G D P}$ in King et al. [2] who use IEA data along with existing time series on energy cost shares and spending for England and the UK, Sweden, and the United States, I conclude that the turn of the 21st Century is, thus far, likely the time of cheapest energy in history. I can make this conclusion because the countries not included in the 44-country aggregate amount to only $5 \%-7 \%$ of world GDP. Thus, even if one assumed the rest of the world were to have rapidly decreased energy expenditures to zero between 1998 and 2002, the newly-estimated $f_{e, G D P}$ would still be at its low point around the year 2000.

My conclusion is based on historical data and does not predetermine that the global $f_{e, \mathrm{GDP}}$ will never be lower in the future. Supplemental Figure S4 calculates $f_{e, G D P}$ to 2014 using data from the BP Statistical Review and indicates that $f_{e, G D P}$ was greater than $6 \%$ for 2010-2014. At the time of this writing, $f_{e, \mathrm{GDP}}$ for the year 2015 will likely be significantly less than from 2006 to 2014 . However, it is safe to say that $f_{e, \mathrm{GDP}}$ will never be zero, as this necessitates free energy (and food) and/or infinite GDP. Thus, one can pose the questions: What future scenarios can decrease $f_{e, \mathrm{GDP}}$ below 1998-2002 levels? What are the implications of no longer decreasing, but perhaps at best only maintaining, the global cost share of energy (and food) that has been so characteristic of transitioning to a modern economy? 


\subsection{Relevance for Internalizing $\mathrm{CO}_{2}$ Emissions}

While the previous discussion is retrospective, here I consider the future possibility of internalizing $\mathrm{CO}_{2}$ emissions into expenditures on energy (see Figure 3). In this section I describe the following rationale. The longer the world waits to reduce annual GHG emissions, the faster the required rate of reductions to stay below a total carbon budget. The faster the reduction in emissions, the higher the rate of investment required to convert to the low-carbon energy system. The larger the investment in the energy system, the more resources are allocated to it, and the higher the $f_{e, \mathrm{GDP}}$ (assuming the energy sector does not go significantly into debt). Consequently, the higher the $f_{e, \mathrm{GDP}}$, the higher the probability of (at least short term) economic recession during the transition.

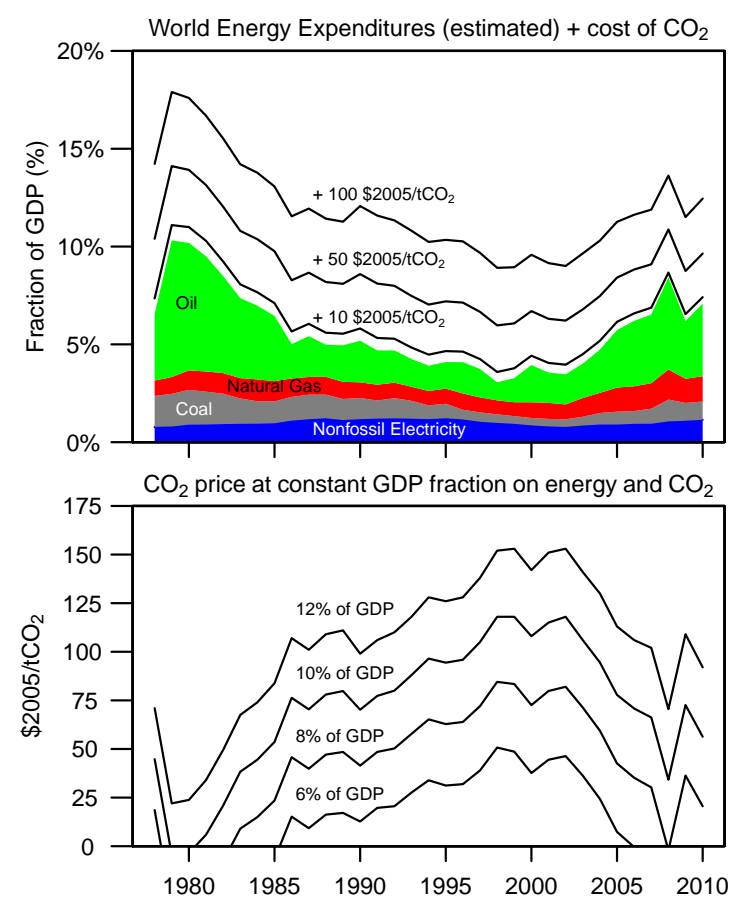

Figure 3. Top: Estimated expenditures on energy $\left(f_{e, \mathrm{GDP}}\right)$ as a fraction of GDP (for the 44 countries in the data set) from 1978 to 2010 including hypothetical costs on $\mathrm{CO}_{2}$ emissions of 10, 50, and 100 $\$ 2005 / \mathrm{tCO}_{2}$. The data for annual $\mathrm{CO}_{2}$ emissions from fossil energy (oil, natural gas, and coal) are from the IEA website [62] Bottom: The $\mathrm{CO}_{2}$ cost that would cause expenditures for both energy and $\mathrm{CO}_{2}$ combined to equal a constant fraction of GDP each year.

Climate models estimate global temperature rises associated with atmospheric GHG concentrations that are a function of cumulative, not annual emissions. This means that a targeted upper bound for future temperature rise relates to an assumed carbon budget for net emissions accumulated over time [63]. Global carbon budget estimates indicate that humans have emitted (since 1870) approximately two-thirds of a global carbon budget to provide a $66 \%$ chance of staying below a $2{ }^{\circ} \mathrm{C}$ increase relative to preindustrial temperatures [64]. This leaves approximately $1200 \mathrm{GtCO}_{2}$ equivalent remaining, or 30 years of current emissions starting from 2015. Emitting more carbon thus lowers the odds that the global temperature rise will stay below $2{ }^{\circ} \mathrm{C}$.

\subsubsection{The Energy Trap}

Because the majority of global primary energy still comes from fossil fuels and approximately $57 \mathrm{t} \mathrm{CO}_{2}$ are emitted per TJ of primary energy [62,65], a rapid reduction in emissions rates translates to a rapid reduction in fossil energy consumption rates and/or substantial investment in $\mathrm{CO}_{2}$ capture and storage systems. Further, it takes energy to manufacture and install renewable energy and low-carbon energy systems. The lower the energy return ratio of low-carbon technologies and the 
faster the low-carbon/renewable energy transition, the higher the increase in short-term energy consumption, $\mathrm{CO}_{2}$ emissions, and costs that are associated with that transition $[66,67]$.

Equations (1)-(3) demonstrate why lower net energy systems make an energy transition more difficult [67]. In these equations, NEER is net external energy ratio equal to energy return on energy invested (EROI) (see King et al. [1] for terminology details), and $\alpha$ is the expansion growth rate of a technology assuming some of the net energy from the energy technology is used to build and install more of that technology. EPBT is the energy payback time, or the time required for the energy technology to generate a quantity of energy equal to the energy required to install the technology (assuming no decomissioning). The other symbols, taken from Kessides and Wade [67] describing power plants, are as follows: $E_{c a p}$ is the energy required for capital manufacturing and installation, $P_{n p}$ is nameplate power capacity, $\phi$ is capacity factor, $h$ is the fraction of gross energy production needed to operate and maintain the technology, $T$ is the lifetime of the technology, $\tilde{t}$ is the construction time to install the technology, and $\beta$ is the chosen fraction of produced energy that is used to construct new power plants. $\beta$ is an economic decision variable, and if $\beta=1$ then all energy from the technology is used to build more of itself.

$$
\begin{gathered}
E R O I=N E E R=\frac{1}{\frac{E_{c a p}}{P_{n p} \phi T}+h} \\
\text { expansion growth rate }=\alpha=\frac{-\left(\frac{1}{\vec{t}}+\frac{1}{T}\right)+\sqrt{\left(\frac{1}{\vec{t}}+\frac{1}{T}\right)^{2}+\frac{4}{\tilde{t}}\left[\frac{\beta \phi(1-h) P_{n p}}{E_{c a p}}-\frac{1}{T}\right]}}{2} \\
\text { energy payback time }=E P B T=\frac{E_{c a p}}{P_{n p} \phi(1-h) T}
\end{gathered}
$$

Imagine a current energy system that is retiring one unit of capacity per year, and that we are used to replacing it with a technology $P_{n p}=1[\mathrm{~W}] ; E_{c a p} 0.01[\mathrm{~J}] ; \phi=0.8, h=0.05 ; T=40[$ year $]$; $\tilde{t}=4[$ year $]$ and $\beta=1$ that has EROI $=$ NEER $=20$, EPBT $=0.013$ years, and $\alpha=8.5$ year $^{-1}$. We consider now replacing the annual retirement with an energy technology that has half the capacity factor, half the lifetime, and requires twice as much energy, $E_{c a p}$, to install the same capacity, $P_{n p}$, as the usual incumbent technology. The new system has NEER $=$ EROI $=19$, approximately equal to the incumbent, but to have the same expansion rate, $\alpha$, it must take only $\tilde{t}=0.8$ years to install. Further, in order to produce the same amount of gross power $\left(=P_{n p} \phi\right)$, we must install twice as many of the new systems each year. Therefore, twice as many new systems at twice the energy to install is four times more energy for installing energy technologies to replace the retiring system. In addition, during installation, there are fewer units of net energy for the energy system to deliver as output to the rest of the economy. Subsequently, energy prices would rise and demand would fall to meet the diminished net supply. This concept has been termed the "energy trap", as noted by Sgouridis [68].

This energy trap scenario is what can happen during a renewable and/or low-carbon transition ${ }^{1}$ [68]. The $\mathrm{CO}_{2}$ emissions associated with a low-carbon transition can act against meeting the annual carbon emissins constraint early in the transition [66]. Some researchers and proponents

1 "For several decades prior to the net energy peak, energy availability is increasing and slowly plateauing creating an institutionalized expectation that it will continue to behave this way. The pace of market-driven ER [renewable energy] investment is accelerating but it proves insufficient to compensate for the reduction following the fossil fuel (actual or climate-constrained) peak. With insufficient renewables built before peaking, the only option for maintaining energy availability post-peak is to raise the investment ratio $\epsilon$ - an action that further reduces the net available energy at the time of such investment. In practice, raising the investment ratio $\epsilon$ after the fact is may be too costly as it effectively increases the perceived energy costs for the entire economy to socially unacceptable levels. A more likely result is a reinforcing cycle of demand destruction (due to high energy costs) and a drop in actual energy investment since, in a situation of dwindling resources, satisfying immediate needs becomes a priority thus diminishing the ability and willingness to invest in renewable resource infrastructure construction. This is the energy trap: the non-renewable resources are allowed to deplete without commensurate investment in renewable resources locking in a lower energy availability state ..." 
believe we can perform a 30-50 year transition to near $100 \%$ use of modern renewable energy without negative impact to economic growth $[69,70]$, but this is simply unknown. It is one thing to know renewable energy systems (e.g., wind and PV solar), powered by primary energy flows, are economic at the margin in today's fossil-dominated economy. It is yet another to model their future complete substitution for stocks of fossil fuels that require minimal investment for storage (e.g., a pile or coal or tank of gasoline). Photovoltaics as a global industry have only recently become net producers of energy during the initial production ramp up [31]. Thus, at this point it is too much of an extrapolation to assume that renewables plus storage (electrochemically, thermally, and as gaseous or liquid fuels such as hydrogen and biofuels) can enable current consumption levels of the developed countries [71,72].

\subsubsection{Internalizing $\mathrm{CO}_{2}$ Emissions into $f_{e, \mathrm{GDP}}$}

Here I contemplate the implications of internalizing $\mathrm{CO}_{2}$ emissions from fossil energy consumption. Data for $\mathrm{CO}_{2}$ emissions for each country come from the IEA (see Supplemental Information for more detail). Internalizing $\mathrm{CO}_{2}$ emissions effectively decreases technology-specific and system-wide power return ratios (PRRs) and energy return ratios (ERRs) due to an emissions penalty (e.g., tax or price) and/or the increased capital and operating investments to reduce $\mathrm{CO}_{2}$ per a given amount of delivered energy. Investments in energy efficiency, for example, can counteract this increase in cost, and efficiency investments become increasingly attractive as energy expenditures increase. Figure 3 (top) presents calculations as if a constant $\mathrm{CO}_{2}$ penalty were internalized in each year. In 1978 each $10 \$ 2005 / \mathrm{tCO}_{2}$ equates to $0.76 \%$ of the GDP in the 44 country data set, and in 2010 it equals $0.56 \%$ of the GDP. Thus, even a low price of $20 \$ 2005 / \mathrm{tCO}_{2}$ in 2010 is larger than the $1 \%$ of global GDP of annual climate-related investments called for in the Stern Review [73].

The internalization of a $\mathrm{CO}_{2}$ tax can be viewed from the standpoint of a threshold $f_{e, \mathrm{GDP}}$ above which the economic growth is severely limited or not possible for the incumbent economic structure. We might not know a precise economically critical $f_{e, \mathrm{GDP}}$ value, that changes over time as the economy undergoes structural changes, but this value certainly exists for a particular economy in its time [14,74]. Just by observing the few data for the modern post-World War II economy, a threshold $f_{e, \mathrm{GDP}}$ likely resides near $8 \%$ (between $6 \%$ and $10 \%$ ) for developed economies. The two post-World War II major worldwide recessions have corresponded with $f_{e, \mathrm{GDP}}>8 \%$, and there is no post-World War II experience of $f_{e, \mathrm{GDP}}>10 \%$ for any extended period of time. Bashmakov [44] explains that this critical threshold value (approximately 11\% for OECD consumer expenditures on energy, not primary energy as in this paper) creates an asymmetric impact on growth. The further $f_{e, \mathrm{GDP}}$ increases above the threshold value, the more the effect starts to dominate the economy and become a binding constraint [44].

Assuming a constant critical value of $f_{e, \mathrm{GDP}}$ that includes a full internalization of $\mathrm{CO}_{2}$ from

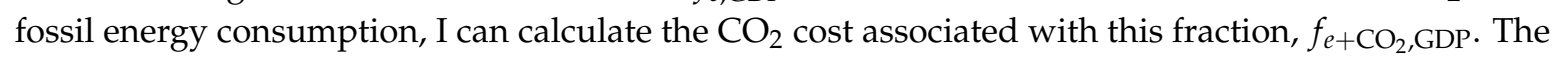

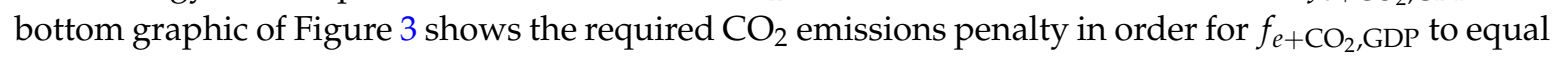
$6 \%, 8 \%, 10 \%$, and $12 \%$ of the 44 country aggregate GDP.

If energy plus $\mathrm{CO}_{2}$ expenditures were to rise toward a growth-limiting $f_{e, \mathrm{GDP}}$, then a market price of $\mathrm{CO}_{2}$ would decrease via feedback to prevent "energy $+\mathrm{CO}_{2}$ " expenditures from breaching a critical $f_{e+\mathrm{CO}_{2}, \mathrm{GDP}}$ that induces recession (see Figure 3). This feedback has been witnessed via the European Trading System (ETS) carbon price. In 2008 the European economy went into recession and has since had low growth. During the same time period the ETS carbon price dropped over $50 \%$ and remains below $10 € / \mathrm{tCO}_{2}$ at time of writing.

In a pre-2008 world, European Union officials assumed that the economy would always grow such that the carbon price would rise to induce new low-carbon investments. An alternative scenario has emerged in which the opposite has happened: a no/low-growth economy has induced a low carbon price. Many have modeled and many hope, but the world has not yet proven, that an 
industrialized world economy can grow and decrease the rate of greenhouse gas (GHG) emissions (or fossil primary energy consumption) in the long run.

\subsubsection{Summary of Relevance for Internalizing $\mathrm{CO}_{2}$ Emissions}

The world is finite, and developed economies already have several near to medium-term structural headwinds to economic growth (e.g., population demographics, inequality, debt accumulation) that are occurring independent from a changing climate. As described by systems approaches, these headwinds are expected to eventually occur on a finite planet [40,41]. As mentioned by Gordon [39] regarding the US, " ... the consequences of environmental regulations and taxes that will make growth harder to achieve than a century ago ...", and thus reducing GHG emissions adds another headwind. The Stern Report (Part III, p. 204) notes that historical reductions in greenhouse gas emissions rates greater than $1 \%$ /year are associated with 'economic recession or upheaval' [63,73]. Anderson and Bows state that reducing emissions by $>3 \%-4 \% /$ year is largely seen as incompatible with economic growth [63]. Further, in one study that relates economic growth to increased use of renewables (one set of mitigation options), Chang et al. [75] analyze OECD countries from 1997-2006. They find that countries with annual GDP growth of $>4.13 \%$ in one year tend to install more renewable energy in the following year whereas lower growth countries are less willing to spend on renewables. This type of insight could partially explain climate treaty success and failure. The Kyoto Protocol was successfully signed in 1997 at the third Conference of Parties (one year after $f_{e, \mathrm{GDP}}=4.0 \%$ ) whereas there was no comprehensive treaty signed at COP 15 (Copenhagen 2009) one year after $f_{e, \mathrm{GDP}}=8.1 \%$. The timing of these meetings relative to energy expenditures is merely coincidence, but perhaps the results are not.

However, sometimes a shift to cheaper fuels happened to coincide with that fuel having lower $\mathrm{CO}_{2}$ emissions (e.g., UK "Dash for Gas" in 1990s [73]), but rarely did total country emissions decrease. One exception is the US post-2008 due to increased activity in horizontal drilling and hydraulic fracturing for hydrocarbons. This drilling activity produced a large quantity of natural gas that has displaced some coal purchased for electricity. This coal displacement coincided with a decrease in total US energy-related $\mathrm{CO}_{2}$ emissions (assuming no fugitive emissions from NG production) from 2007 to 2012 as well as a major economic downturn. However, the increased natural gas production was primarily driven by technological, financial, and other economic factors unrelated to policy goals for reducing GHG emissions. Further, globalization significantly disconnects the location of energy consumption (and GHG emissions) from that of consumption of goods and services [12]. Thus, unless a country halts international trade of all goods and services, it is difficult to claim all absolute GHG reductions from energy from within the country's borders.

Suffice it to say, predicting long-term economic growth, with or without GHG mitigation, is difficult to impossible. Most studies promoting renewable energy and/or the reduction of GHG emissions project economic growth and rapid GHG emissions reductions can happen simultaneously and into the future as far as they simulate [76]. Here I use information from the Intergovernmental Panel on Climate Change (IPCC) Fifth Assessment Report (AR5) as an example. The IPCC AR5 summarizes that GHG emissions reductions $\geq 3 \%$ /year are needed by 2030 and can accompany economic growth [77]. The IPCC is informed by many models running baseline scenarios (those without additional mitigation efforts) from Integrated Assessment Models (IAMs) and other analyses. These baseline scenarios used by the IPCC indicate consumption grows anywhere from $300 \%$ to more than $900 \%$ between 2010 and 2100 . When simulating idealized mitigation conditions, the IPCC estimates "...that reaching about 450 ppm $\mathrm{CO}_{2}$ eq by 2100 would entail global consumption losses of. . . $3 \%$ to $11 \%$ in 2100 " [78] or GDP losses of typically less than $10 \%$ in 2100 .

Thus, most simulations show mitigation costs as trivial compared to gains in economic growth, and within the noise of the accuracy of the models. No matter what the investment in the energy system or the level of climate damages, the models simulate that economy will always grow. Stern [79] and Pindyck [80] point out how much IAMs are likely to underestimate damages and 
overestimate economic growth. One major reason is that IAMs often incorporate an exogenous assumption of constant annual rate of increase in total factor productivity (TFP) that is independent of energy-related factors (see Figure A.II.1 of [81]). Thus, the normal IAM assumption is inadequate because it presents the case to policy makers that even dramatic increases in energy investment for a renewable energy transition and/or climate change mitigation don't affect TFP and hence economic growth $[79,80]$.

In addition, as pointed out by Loftus et al. [76], many low-carbon scenarios assume an annual decrease in energy intensity, $\epsilon=\mathrm{TPES} / \mathrm{GDP}$, that is either faster than historical trends $(\sim-0.8 \%$ /year from 1970-2010 [77]) or is sustained at decline rates of $-1.5 \% /$ year to $-2 \% /$ year that have only been temporarily achieved since 1970 (e.g., in the early 1980s and late 1990s/early 2000s). The mean (or "default") of the baseline scenarios as used by the IPCC AR5 assume global energy intensity changes of approximately $-1.0 \%$ /year to $-1.7 \%$ /year (e.g., Figure 6.17 Chapter 6-Assessing Transformation Pathways [78]).

If TFP and declines in energy intensity are overestimated, the subsequent calculations of the social cost of carbon (SCC) will be understated. Stern [79] notes that this effectively leads one to conclude that high concentration levels (e.g., 650 ppm $\mathrm{CO}_{2}$ eq) are acceptable. However, as previously discussed, results in this paper and other research show that energy resource and technology characteristics (e.g., conversion efficiency) can partially or largely describe TFP via impact on energy cost share $[8,10,18]$. TFP is negatively affected by higher $f_{e, \text { GDP }}$ that is itself a factor of the energy technologies and the rate of low-carbon energy transformation that IAMs are meant to simulate. Hence, if we invest in energy systems with worse biophysical and economic qualities (e.g., lower net energy, higher cost) and at a higher rate, we should expect lower TFP and hence lower economic growth. In addition to optimistic assumptions about TFP (even if assumed lower than historical values) and energy intensity, both Stern [79] and Pindyck [80] indicate that climate models grossly underestimate climate damages at high atmospheric concentration levels. Thus, those authors would argue that estimates for the social cost of carbon (SCC) are also too small.

Here I show an example calculation indicating that the future annual emissions cost ranges from the IPCC translate to $1 \%-2 \%$ of GDP-enough to potentially increase $f_{e+\mathrm{CO}_{2}, \mathrm{GDP}}$ from $6 \%-7 \%$ to above $8 \%$, or from a condition associated with growth to one associated with recession. The IPCC reports that global carbon prices for a target $430-530 \mathrm{ppm} \mathrm{CO}_{2}$ eq would be near $1500 \$ 2010 / \mathrm{tCO}_{2}$ (150-6000 $\$ 2010 / \mathrm{tCO}_{2}$ ) in 2100 (Figure 6.21 [78]). The median net present value (NPV) of the price of $\mathrm{CO}_{2}$ from these simulations is approximately $36 \$ 2010 / \mathrm{tCO}_{2}\left(20\right.$ to $55 \$ 2010 / \mathrm{tCO}_{2}$ at $25 \%-75 \%$ range, Figure 6.21 [78]). Applying this median NPV price of carbon $\left(=33 \$ 2005 / \mathrm{tCO}_{2}\right)$ to the 2010 emissions of the 44-country IEA data set (as in Figure 3) translates to $940 \$ 2005$ billion (=1.8\% of GDP), or $f_{e+\mathrm{CO}_{2}, \mathrm{GDP}}=6.9 \%+1.8 \%=8.7 \%$. Thus, the range of $\mathrm{CO}_{2}$ price translates to $1 \%-2 \%$ of GDP. If $f_{e, \mathrm{GDP}}$ is already greater than $6 \%-7 \%$ of GDP (as it was in 1978-1985, 2008, 2010, and likely also during 2011-2014, see Supplemental Figure S4) then $f_{e+\mathrm{CO}_{2}, \mathrm{GDP}}$ at these $\mathrm{CO}_{2}$ prices will be $7 \%-9 \%$ of GDP, at a possible threshold above which there has historically been recession.

Climate policy might have no choice but to accept decreasing rates of GHG emissions along with a declining economy. The reasoning of this previous sentence is not based upon the uniqueness of investing in climate change mitigation. Rather, the reasoning derives from the mathematical premise of Pareto optimality [82]. If one has optimized one objective (e.g., economic growth) without harming a second objective (e.g., GHG emissions), and also optimized the second objective without harming the first, it is then mathematically impossible to simultaneously further optimize one objective without hurting the other. Thus, it is possible that the world economy can reach a time where there is a necessary tradeoff between maximizing economic growth and minimizing GHG emissions (or any number of other socio-economic objectives). With respect to a tradeoff of economy and GHG, I do not claim that the world economy is currently at a point of Pareto optimality, that the world will ever will be at such a point, or that we will ever know if we reside at such optimality. I only state that this tradeoff of economic growth versus climate mitigation is real. 


\subsection{Broader Considerations for Policy and Future Research}

It is important to note that in this paper I am not advocating any specific policy action. I also advocate neither that the economy should grow at the expense of the environment nor that the economy should shrink to reduce environmental impacts and risks. Most policies do not advocate either of these paradigms, but instead state that we have and always will be able to both grow the economy and reduce environmental impacts. I do, however, suggest that it is very constructive to better understand if/when our energy resources and technologies are incapable of maintaining or expanding the size and complexity of our existing economy and social structure. While the Estimated energy expenditures from Part 2 [2] of this series stops at 2010, global oil prices remained high through mid-2014 before dropping over $50 \%$ to below $\$ 50 /$ BBL as of October 2015 (the time of writing). Figure S4 of the Supplemental Information compares my Estimated energy expenditures to a quick estimate of global expenditures for oil, natural gas, and coal using data from BP's Statistical Review of World Energy. Energy expenditures through mid-2014 likely remained at $f_{e, \mathrm{GDP}}$ of $7 \%-8 \%$, and during this time developed economy growth was near stagnant.

While I believe the consideration of expenditures on energy is valuable for understanding the role of energy in the macroeconomy, the results of this paper are limited in many respects, not all of which can be discussed here. I have based the analyses on energy prices, and not costs, such that the net energy of the energy sector itself is actually higher than what the cost share calculation suggests (net power ratio based on intermediate spending and gross capital formation, $\mathrm{NPR}_{\mathrm{economic}} \sim f_{e, \mathrm{GDP}}^{-1}$, see Part 2 [2]). In other words, market prices (theoretically) represent the marginal cost producer, and they enable low-cost energy producers to accumulate rents as profits. On the other hand, the world has slowly adjusted to these rents such that even governments of energy exporting nations that subsidize their citizens' energy consumption might not be able to easily adjust to lower market prices if they require a high market energy price to balance the national budget [83].

Also, there are many growth "headwinds" for the US and other developed economies: demography, education, inequality, globalization, energy/environment, and the overhang of consumer and government debt [39]. This paper describes only trends in the "energy/environment" headwind, but from a systems viewpoint we must understand how to include energy along with other drivers such as demographic shifts and population as well as debt accumulation. A major part of the industrial transition (e.g., per Figure 1) is due to declining the role of food and biomass as input to industrial output, in turn due to fossil-fueled machinery replacing human and animal labor. Thus, if $f_{e, \mathrm{GDP}}$ including food is decreasing fast enough (e.g., NPR economic is increasing fast enough), perhaps an increasing population is possible that, in turn, allows higher total throughput. At too low

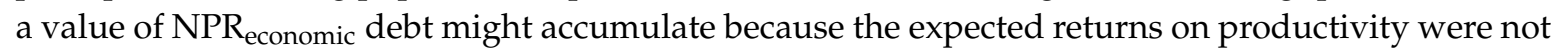
realized. In effect, one might interpret some debt defaults as the result of too many previous claims on actual future energy production (kudos to Nate Hagens for this viewpoint). Future research should also focus on understanding how to include debt as an additional economic metric to relate to net energy metrics.

Expected economic returns are partly based on historical experience, such that if the energy cost trends underlying that experience no longer continue, then economic history and future scenarios must be interpreted in this context. Future economic scenarios largely focus on internalizing energy-related GHG emissions, and we need to need to complement economic modeling approaches with biophysical approaches. One way is to increase understanding of the feedbacks among economic growth, $f_{e, \mathrm{GDP}}$, and the quantity of resource allocation to the energy sector. Since World War II, the developed world was in recession during both instances of $f_{e, \mathrm{GDP}}>8 \%$. The faster and further one transitions from the incumbent energy infrastructure, the higher $f_{e, \mathrm{GDP}}$ becomes (at least in the short run) due to increased allocation of labor, capital, and natural resources to energy production. This rationale is akin to the IPCC's statement that "Estimates of the aggregate economic costs of mitigation vary widely, but increase with stringency of mitigation (high confidence)" [77]. This negative feedback of energy cost share on economic growth raises the question as to whether world citizens will choose 
to charge themselves for $\mathrm{CO}_{2}$ emissions to the extent the choice might cause a recession due to the costs of rapidly replacing energy infrastructure and/or reduced consumption. Just as central banks adjust interest rates, we could choose to throttle the economy by directly or indirectly increasing $\mathrm{CO}_{2}$ prices or taxes. If at any time interest rates and $\mathrm{CO}_{2}$ prices are both near zero (as is largely the case today) it would indicate that economic growth is prioritized. If socioeconomic conditions are acceptable, then we could choose to increase $\mathrm{CO}_{2}$ prices and/or interest rates. Theoretically, policy could target a $\mathrm{CO}_{2}$ price feedback to target a GDP growth rate, including zero growth.

\section{Conclusions}

The calculation of expenditures on energy, combined with long-term historical assessments of expenditures on energy, indicates that the turn of the 21st Century is, thus far, likely the time of cheapest worldwide energy in the history of mankind. Preindustrial United Kingdom and Sweden spent over four times more on energy than they do today. Thus, the trend during industrialization of decreasing expenditures on energy, measured relative to GDP $f_{e, G D P}$, is unquestionable. Has the long-term trend of decreasing $f_{e, \mathrm{GDP}}$ stopped? To answer this question we need biophysical and economic models that can quantify $f_{e, \mathrm{GDP}}$ from the pre-industrial economy to today as well as for future scenarios. Energy expenditures cannot be zero, and $f_{e, \mathrm{GDP}}$ cannot go to zero. These conditions necessitate infinite GDP or no investment to deliver energy-two impossibilities. However, it is not necessary to solely minimize $f_{e, G D P}$ to achieve sufficient livelihoods as other goals (e.g., environmental sustainability) are important to consider even if the feedbacks increase $f_{e, \mathrm{GDP}}$ in the short and/or long-term.

I did not perform a rigorous econometric analysis of the energy cost share data in relation to GDP and total factor productivity. However, my correlation analysis shows that energy cost share, $f_{e, \mathrm{GDP}}$, plays a significant role in describing global total factor productivity and GDP. The correlation analysis is consistent with findings in the literature that include quality-adjusted energy as a factor of economic production along with capital and labor. The quality adjustments are usually some metric of energy preference (e.g., weighted by relative prices of commodities), cost share-as in this paper, or a technological characteristic such as exergy or conversion efficiency from energy to energy services or useful work.

In addition to putting global expenditures on energy in historical perspective, I explain why internalizing $\mathrm{CO}_{2}$ is so challenging. If internalizing the cost of $\mathrm{CO}_{2}$ emissions from fossil energy in the year of cheapest energy (1998), I find that adding $100 \$ 2005 / \mathrm{tCO}_{2}$ to $f_{e, \mathrm{GDP}}$ would have caused "energy $+\mathrm{CO}_{2}$ " expenditures to rise to $9 \%$ of world GDP. Modern developed economies have no experience of continued economic growth coinciding with this level of expenditures on energy. In 2010 , it would have taken only $38 \$ 2005 / \mathrm{tCO}_{2}$ to reach $f_{e+\mathrm{CO}_{2}, \mathrm{GDP}}=9 \%$, a much lower price than required to induce large scale reductions in emissions.

Acknowledgments: This work was partially supported by, and the authors thank, the Jackson School of Geosciences Energy Theme at The University of Texas at Austin. The author also thanks Roger Fouquet for his correspondence regarding historical England/UK data on expenditures on energy for energy services.

Conflicts of Interest: The authors declare no conflict of interest.

\section{References}

1. King, C.W.; Maxwell, J.P.; Donovan, A. Comparing world economic and net energy metrics, Part 1: Price Perspective. Energies 2015, 8, 12949-12974.

2. King, C.W.; Maxwell, J.P.; Donovan, A. Comparing world economic and net energy metrics, Part 2: Expenditures Perspective. Energies 2015, 8, 12975-12996.

3. Solow, R. A Contribution to the Theory of Economic-Growth. Q. J. Econ. 1956, 70, 65-94.

4. Hamilton, J. Historical Oil Shocks. In Routledge Handbook of Major Events in Economic History; Parker, R.E., Whaples, R.M., Eds.; Routledge: New York, NY, USA, 2013; p. 239265. 
5. Ayres, R.; Voudouris, V. The economic growth enigma: Capital, labour and useful energy? Energy Policy 2014, 64, 16-28.

6. Voudouris, V.; Ayres, R.; Serrenho, A.C.; Kiose, D. The economic growth enigma revisited: The EU-15 since the 1970s. Energy Policy 2015, doi:10.1016/j.enpol.2015.04.027.

7. Kander, A.; Stern, D.I. Economic growth and the transition from traditional to modern energy in Sweden. Energy Econ. 2014, 46, 56-65.

8. Kümmel, R. The Second Law of Economics: Energy, Entropy, and the Origins of Wealth; Springer: Berlin, Germany, 2011.

9. Hall, C.A.S.; Klitgaard, K.A. Energy and the Wealth of Nations: Understanding the Biophysical Economy, 1st ed.; Springer: Berlin, Germany, 2012.

10. Ayres, R.U. Sustainability economics: Where do we stand? Ecol. Econ. 2008, 67, 281-310.

11. Brown, J.H.; Burnside, W.R.; Davidson, A.D.; Delong, J.R.; Dunn, W.C.; Hamilton, M.J.; Mercado-Silva, N.; Nekola, J.C.; Okie, J.G.; Woodruff, W.H.; et al. Energetic Limits to Economic Growth. BioScience 2011, 61, 19-26.

12. Peters, G.P.; Hertwich, E.G. CO2 embodied in international trade with implications for global climate policy. Environ. Sci. Technol. 2008, 42, 1401-1407.

13. Acemoglu, D.; Robinson, J.A. Why Nations Fail: The Origins of Power, Prosperity, and Poverty; Crown Business: New York, NY, USA, 2012.

14. Tainter, J. The Collapse of Complex Societies; Cambridge University Press: Cambridge, UK, 1988.

15. Tainter, J.A. Energy, complexity, and sustainability: A historical perspective. Environ. Innov. Soc. Transit. 2011, 1, 89-95.

16. Tainter, J.A. Energy and Existential Sustainability: The Role of Reserve Capacity. J. Environ. Account. Manag. 2013, 1, 213-228.

17. Turchin, P.; Nefedov, S.A. Secular Cycles; Princeston University Press: Princeston, NJ, USA, 2009.

18. Ayres, R.U.; Warr, B. Accounting for growth: The role of physical work. Struct. Chang. Econ. Dyn. 2005, 16, 181-209.

19. Stern, D.; Kander, A. The Role of Energy in the Industrial Revolution and Modern Economic Growth; Technical Report; The Australian National University: Canberra, Australia, 2011.

20. Stern, D.I.; Kander, A. The Role of Energy in the Industrial Revolution and Modern Economic Growth. Energy J. 2012, 33, doi:10.5547/01956574.33.3.5.

21. Hamilton, J. Causes and Consequences of the Oil Shock of 2007-08. In Brookings Papers on Economic Activity; Romer, D., Wolfers, J., Eds.; Brookings Institution Press: Washington, DC, USA, 2009; p. 69.

22. Hall, C.A.S.; Cleveland, C.J.; Kaufmann, R.K. Energy and Resource Quality: ThE Ecology of the Economic Process; Wiley: New York, NY, USA, 1986.

23. Hall, C.A.S.; Balogh, S.; Murphy, D.J.R. What is the Minimum EROI that a Sustainable Society Must Have? Energies 2009, 2, 25-47.

24. King, C.W. Energy intensity ratios as net energy measures of United States energy production and expenditures. Environ. Res. Lett. 2010, 5, 044006.

25. Brandt, A.R. Converting oil shale to liquid fuels: Energy inputs and greenhouse gas emissions of the Shell in situ conversion process. Environ. Sci. Technol. 2008, 42, 7489-7495.

26. Brandt, A.R. Converting Oil Shale to Liquid Fuels with the Alberta Taciuk Processor: Energy Inputs and Greenhouse Gas Emissions. EnergyFuels 2009, 23, 6253-6258.

27. Brandt, A.R.; Englander, J.; Bharadwaj, S. The energy efficiency of oil sands extraction: Energy return ratios from 1970 to 2010. Energy 2013, 55, 693-702, doi:10.1016/j.energy.2013.03.080.

28. Guilford, M.C.; Hall, C.A.S.; O'Connor, P.; Cleveland, C.J. A New Long Term Assessment of Energy Return on Investment (EROI) for US Oil and Gas Discovery and Production. Sustainability 2011, 3, 1866-1887.

29. Farrell, A.E.; Plevin, R.J.; Turner, B.T.; Jones, A.D.; O'Hare, M.; Kammen, D.M. Ethanol can contribute to energy and environmental goals. Science 2006, 311, 506-508.

30. Raugei, M.; Fullana-i-Palmer, P.; Fthenakis, V. The energy return on energy investment (EROI) of photovoltaics: Methodology and comparisons with fossil fuel life cycles. Energy Policy 2012, 45, 576-582, doi:10.1016/j.enpol.2012.03.008. 
31. Dale, M.; Benson, S.M. Energy Balance of the Global Photovoltaic (PV) Industry-Is the PV Industry a Net Electricity Producer? Environ. Sci. Technol. 2013, 47, 3482-3489. Available online: http://pubs.acs.org/doi/pdf/10.1021/es3038824 (accessed on 3 March 2015).

32. Fthenakis, V.M.; Kim, H.C. Photovoltaics: Life-cycle analyses. Sol. Energy 2011, 85, 1609-1628.

33. Zhang, Y.; Colosi, L.M. Practical ambiguities during calculation of energy ratios and their impacts on life cycle assessment calculations. Energy Policy 2013, 57, 630-633.

34. Bullard, C.W., III; Herendeen, R.A. The energy cost of goods and services. Energy Policy 1975, 3, 268-278, doi:10.1016/0301-4215(75)90035-X.

35. Costanza, R. Embodied Energy and Economic Valuation. Science 1980, 210, 1219-1224.

36. King, C.W.; Hall, C.A.S. Relating Financial and Energy Return on Investment. Sustainability 2011, 3, 1810-1832.

37. Henshaw, P.F.; King, C.; Zarnikau, J. System Energy Assessment (SEA), Defining a Standard Measure of EROI for Energy Businesses as Whole Systems. Sustainability 2011, 3, 1908-1943.

38. Summers, L.H. Have We Entered an Age of Secular Stagnation? IMF Econ. Rev. 2015, 63, 277-280.

39. Gordon, R.J. Is US Economic Growth Over? Faltering Innovation Confronts the Six Headwinds; NBER Working Paper No. 18315; National Bureau of Economic Research (NBER): Cambridge, MA, USA, 2012.

40. Meadows, D.H.; Meadows, D.L.; Randers, J.; Behrens, W.W.I. Limits to Growth: A Report for the Club of Rome's Project on the Predicament of Mankind; Universe Books: New York, NY, USA, 1972.

41. Meadows, D.H.; Randers, J.; Meadows, D.L. Limits to Growth: The 30-Year Update; Chelsea Green Publishing: White River Junction, VT, USA, 2004.

42. Kilian, L. Oil Price Shocks, Monetary Policy and Stagflation; In proceedings of Conference on Inflation in an Era of Relative Price Shocks, Sydney, Australia, 17-18 August 2009. Available online: http://www-personal.umich.edu/ lkilian/rbakilianpub.pdf (accessed on 3 March 2015).

43. Kopits, S. Oil: What Price can America afford? Available online: http://www.oilandgasinvestor.com/ oil-what-price-can-america-afford-465256 (accessed on 3 March 2015).

44. Bashmakov, I. Three laws of energy transitions. Energy Policy 2007, 35, 3583-3594.

45. Kalimeris, P.; Richardson, C.; Bithas, K. A meta-analysis investigation of the direction of the energy-GDP causal relationship: implications for the growth-degrowth dialogue. J. Clean. Prod. 2014, 67, 1-13.

46. Cleveland, C.J.; Kaufmann, R.K.; Stern, D.I. Aggregation and the role of energy in the economy. Ecol. Econ. 2000, 32, 301-317.

47. Odum, H.T. Environmental Accounting: Energy and Environmental Decision Making; John Wiley \& Sons, Inc.: New York, NY, USA, 1996.

48. Campbell, D.E.; Lu, H.; Walker, H.A. Relationships among the Energy, Emergy and Money Flows of the United States from 1900 to 2011. Front. Energy Res. 2014, 2, doi:10.3389/fenrg.2014.00041.

49. Stern, D.I. Energy and economic growth in the USA: A multivariate approach. Energy Econ. 1993, 15, 137-150, doi:10.1016/0140-9883(93)90033-N.

50. Cleveland, C.J.; Costanza, R.; Hall, C.A.S.; Kaufmann, R.K. Energy and the US Economy: A Biophysical Perspective. Science 1984, 225, 890-897.

51. Stern, D.I. The role of energy in economic growth. Ann. N. Y. Acad. Sci. 2011, 1219, $26-51$.

52. The Conference Board. Total Economy Database, 2013. Available online: http://www.conference-board. org/data/economydatabase (accessed 17 December 2013).

53. Organization for Economic Co-operation and Development. OECD. Stat Database, 2013. Available online: http:/ / stats.oecd.org (accessed 17 December 2013).

54. Fouquet, R. Heat, Power, and Light: Revolutions in Energy Services; Edward Elgar Publishing Limited: Northampton, MA, USA, 2008.

55. Fouquet, R. The slow search for solutions: Lessons from historical energy transitions by sector and service. Energy Policy 2010, 38, 6586-6596, doi:10.1016/j.enpol.2010.06.029.

56. Fouquet, R. Divergences in Long-Run Trends in the Prices of Energy and Energy Services. Rev. Environ. Econ. Policy 2011, 5, 196-218, doi:10.1093/reep/rer008. Data available for download: http:/ / www.lse.ac.uk/GranthamInstitute/publication/data-set-on-the-price-of-energy-and-energyservices-1700-2010-2/ (accessed on 3 March 2015). 
57. Fouquet, R. Long run demand for energy services: income and price elasticities over 200 years. Rev. Environ. Econ. Policy 2014, 8, 186-207. Data available for download: http://www.lse.ac.uk/ GranthamInstitute/publication/divergences-in-long-run-trends-in-the-prices -of-energy-and-energy-services/ (accessed on 3 March 2015).

58. Broadberry, S.; Campbell, B.; Klein, A.; Overton, M.; van Leeuwen, B. British Economic Growth, 1270-1870: An Output-Based Approach; School of Economics, University of Kent, UK, 2012.

59. Mitchell, B.R. British Historical Statistics; Cambridge University Press: Cambridge, UK, 1988.

60. United States Bureau of Economic Analysis (BAE). Personal Consumption Expenditures by Major Type of Product, Table 2.3.5 BAE: Washington, DC, USA, 29 August 2012.

61. Aucott, Michael and Hall, Charles. Does a Change in Price of Fuel Affect GDP Growth? An Examination of the U.S. Data from 1950-2013. Energies 2014, 7, 6558-6570.

62. IEA. Carbon dioxide emissions from fossil fuel combustion 2014. Available online: http://www.iea.org/media/freepublications/stats/_CO2_Emissions_From_Fuel_Combustion_ Highlights_2014.xls (accessed on 6 July 2015).

63. Anderson, K.; Bows, A. Beyond "dangerous" climate change: Emission scenarios for a new world. Philos. Trans. R. Soc. A Math. Phys. Eng. Sci. 2011, 369, 20-44. Available online: http:/ / rsta.royalsocietypublishing.org/content/369/1934/20.full.pdf+html (accessed on 3 March 2015).

64. Friedlingstein, P.; Andrew, R.M.; Rogelj, J.; Peters, G.P.; Canadell, J.G.; Knutti, R.; Luderer, G.; Raupach, M.R.; Schaeffer, M.; van Vuuren, D.P.; et al. Persistent growth of CO2 emissions and implications for reaching climate targets. Nat. Geosci. 2014, 7, 709-715.

65. IEA. Key World Energy Statistics 2014. Available online: http://www.iea.org/publications/ freepublications/publication/keyworld2014.pdf (accessed on 3 October 2015).

66. Utamura, M. Analytical model of carbon dioxide emission with energy payback effect. Energy 2005, 30, 2073-2088.

67. Kessides, I.N.; Wade, D.C. Towards a sustainable global energy supply infrastructure: Net energy balance and density considerations. Energy Policy 2011, 39, 5322-5334, doi:10.1016/j.enpol.2011.05.032.

68. Sgouridis, S. Defusing the energy trap: The potential of energy-denominated currencies to facilitate a sustainable energy transition. Front. Energy Res. 2014, 2, doi:10.3389/ fenrg.2014.00008.

69. Jacobson, M.Z.; Delucchi, M.A. Providing all global energy with wind, water, and solar power, Part I: Technologies, energy resources, quantities and areas of infrastructure, and materials. Energy Policy 2011, 39, 1154-1169.

70. Delucchi, M.A.; Jacobson, M.Z. Providing all global energy with wind, water, and solar power, Part II: Reliability, system and transmission costs, and policies. Energy Policy 2011, 39, 1170-1190.

71. Lloyd, B.; Forest, A.S. The transition to renewables: Can PV provide an answer to the peak oil and climate change challenges? Energy Policy 2010, 38, 7378-7394.

72. Moriarty, P.; Honnery, D. What energy levels can the Earth sustain? Energy Policy 2009, 37, 2469-2474.

73. Stern, N. The Economics of Climate Change: The Stern Review; Cambridge University Press: Cambridge, UK, 2006.

74. Tainter, J.A.; Patzek, T.W. Drilling Down: The Gulf Oil Debacle and Our Energy Dilemma; Springer: Berlin, Germany, 2012.

75. Chang, T.H.; Huang, C.M.; Lee, M.C. Threshold effect of the economic growth rate on the renewable energy development from a change in energy price: Evidence from OECD countries. Energy Policy 2009, 37, 5796-5802.

76. Loftus, P.J.; Cohen, A.M.; Long, J.C.S.; Jenkins, J.D. A critical review of global decarbonization scenarios: what do they tell us about feasibility? Wiley Interdiscip. Rev. Clim. Chang. 2015, 6, 93-112.

77. Intergovernmental Panel on Climate Change (IPCC). Climate Change 2014: Synthesis Report. Contribution of Working Groups I, II and III to the Fifth Assessment Report of the Intergovernmental Panel on Climate Change; IPCC: Geneva, Switzerland, 2014; p. 151. 
78. Clarke, L.; Jiang, K.; Akimoto, K.; Babiker, M.; Blanford, G.; Fisher-Vanden, K.; Hourcade, J.C.; Krey, V.; Kriegler, E.; Löschel, A.; et al. Assessing Transformation Pathways. In Climate Change 2014: Mitigation of Climate Change. Contribution of Working Group III to the Fifth Assessment Report of the Intergovernmental Panel on Climate Change; Edenhofer, O., Pichs-Madruga, R., Sokona, Y., Farahani, E., Kadner, S., Seyboth, K., Adler, A., Baum, I., Brunner, S., Eickemeier, P., et al., Eds.; Cambridge University Press: Cambridge, UK, 2014.

79. Stern, N. The Structure of Economic Modeling of the Potential Impacts of Climate Change: Grafting Gross Underestimation of Risk onto Already Narrow Science Models. J. Econ. Lit. 2013, 51, 838-859, doi:10.1257/jel.51.3.838.

80. Pindyck, R.S. Climate Change Policy: What Do the Models Tell Us? J. Econ. Lit. 2013, 51, 860-872.

81. Krey, V. Annex II: Metrics \& Methodology. In Climate Change 2014: Mitigation of Climate Change. Contribution of Working Group III to the Fifth Assessment Report of the Intergovernmental Panel on Climate Change; Edenhofer, O., Pichs-Madruga, R., Sokona, Y., Farahani, E., Kadner, S., Seyboth, K., Adler, A., Baum, I., Brunner, S., Eickemeier, P., et al., Eds.; Cambridge University Press: Cambridge, UK, 2014.

82. Papalambros, P.Y.; Wilde, D.J. Principles of Optimal Design: Modeling and Computation, 2nd ed.; Cambridge University Press: New York, NY, USA, 2000.

83. Aissaoui, A. Fiscal Break-Even Prices Revisited: What More Could They Tell Us about OPEC Policy Intent? Arab Petroleum Investments Corporation Economic Commentary, 7 (8-9), APICORP: Dammam, Saudi Arabia, 2012. Available online: http://www.apic.com/ Research/Commentaries/Commentary_V7_N8-9_2012.pdf (accessed on 3 March 2015).

(C) 2015 by the author; licensee MDPI, Basel, Switzerland. This article is an open access article distributed under the terms and conditions of the Creative Commons by Attribution (CC-BY) license (http://creativecommons.org/licenses/by/4.0/). 